\title{
Review of Ingot Niobium as a Material for Superconducting Radiofrequency Accelerating Cavities
}

\author{
P. Kneisel, ${ }^{1 *}$ G. Ciovati, ${ }^{1}$ P. Dhakal, ${ }^{1}$ K. Saito, ${ }^{2}$ W. Singer, ${ }^{3}$ \\ X. Singer, ${ }^{3}$ and G. R. Myneni ${ }^{1 \#}$ \\ ${ }^{1}$ Jefferson Lab, Newport News, VA 23606, USA \\ ${ }^{2}$ Michigan State University, East Lansing, MI 48824, USA \\ ${ }^{3}$ DESY, Notkestrasse 85, D-22607 Hamburg, Germany
}

\begin{abstract}
$\underline{\text { Abstract }}$
As a result of collaboration between Jefferson Lab and niobium manufacturer Companhia Brasileira de Metalurgia e Mineração (CBMM), ingot niobium was explored as a possible material for superconducting radiofrequency (SRF) cavity fabrication. The first single cell cavity from large-grain high purity niobium was fabricated and successfully tested at Jefferson Lab in 2004. This work triggered research activities in other SRF laboratories around the world. Large-grain (LG) niobium became not only an interesting alternative material for cavity builders, but also material scientists and surface scientists were eager to participate in the development of this technology. Many single cell cavities made from material of different suppliers have been tested successfully and several multi-cell cavities have shown performances comparable to the best cavities made from standard fine-grain niobium. Several 9-cell cavities fabricated by Research Instruments and tested at DESY exceeded the best performing fine grain cavities with a record accelerating gradient of $E_{a c c}=45.6 \mathrm{MV} / \mathrm{m}$. The quality factor of those cavities was also higher than that of fine-grain (FG) cavities processed with the same methods. Such performance levels push the state-of-the art of SRF technology and are of great interest for future accelerators. This contribution reviews the development of ingot niobium technology and highlights some of the differences compared to standard FG material and opportunities for further developments.
\end{abstract}

PACS numbers: 84.40.-x, 84.71.-b 


\section{INTRODUCTION}

Niobium was introduced as material for superconducting accelerating cavities in 1967, replacing the lead-plated copper cavities, which had been used at High Energy Physics Lab (HEPL), Stanford University, Kernforschungszentrum Karlsruhe (KfK), Brookhaven National Lab (BNL) and CERN. Initially, the electrolytic deposition of niobium from a salt bath onto a copper substrate was used [1]; however, because of the high deposition temperature of $740{ }^{\circ} \mathrm{C}$ the layers were contaminated by diffused $\mathrm{Cu}$ ions. Later, Siemens AG succeeded to fabricate a $\mathrm{TE}_{011} \mathrm{X}$-band cavity by this method, but with low $Q_{0}$ values [2]. At Stanford University, which was the pioneering laboratory in the development of niobium cavities, the first results were achieved in 1968. Starting with vapor deposition of thin layers and electro-deposition the research quickly turned to using solid reactor grade niobium and machining X-band cavities from ingot material $[3,4,5$, 6]. This route was also taken at Siemens AG [2] and at KfK for R\&D cavities and separator modules [7]. Figure 1 shows some examples of cavities made from ingot material or electro-deposition.
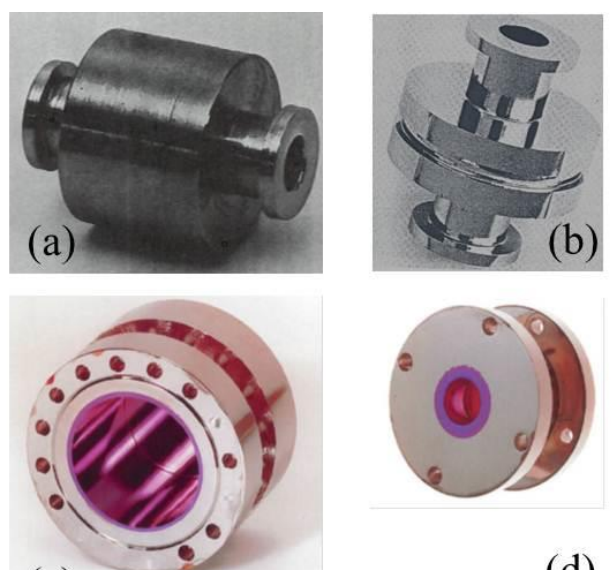

(c)

(d)
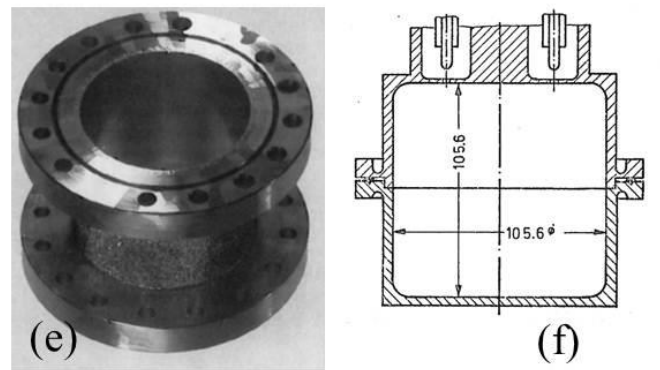

(f)

Fig. 1. Niobium Cavities from Ingot Niobium: (a) and (b) are manufactured at HEPL, $\mathrm{TM}_{010}$ cavity [4, 6], (c) $\mathrm{TE}_{011}$ cavity [2], (d) $\mathrm{TM}_{010}$ cavity fabricated and anodized at Siemens (e) manufactured by electro-deposition at Siemens and (f) is the schematic of a $\mathrm{TM}_{011}$ cavity manufactured at KfK [7].

These cavities from ingot, reactor grade (residual resistivity ratio, RRR 40) material performed well with peak surface magnetic fields as high as $107 \mathrm{mT}$ [6] and 159 
mT [8], respectively. Following the excellent results obtained with X-band cavities at HEPL, multi-cell L-band cavities for the Superconducting Linear Accelerator were designed and fabricated. Results from several cavities made from hydro-formed sheet metal discs were reported in ref. [9]. The manufacturing technology developed at HEPL in the 1970s used deep drawing of half cells, machining to final dimensions and electron beam welding at the equators and irises; it was copied by every cavity fabricator since then with only minor modifications such as elimination of the final machining step; niobium sheet metal became the material of choice. Since then several accelerator projects rely on SRF cavities and the fabrication technology based on high purity $\mathrm{Nb}$ sheet or sputtered $\mathrm{Nb}$ on copper cavities.

The high purity $\mathrm{Nb}$ sheets which are used in the standard fabrication of SRF cavities have a grain size of $\sim 50 \mu \mathrm{m}(\mathrm{ASTM} \geq 5)$. A small and uniform grain size was considered to be a necessary requirement for good formability of polycrystalline material to avoid an excessive roughness of the surface after deep-drawing (having the appearance of an "orange peel"), resulting from the non-uniform deformation of large grains with different crystalline orientation. The production of fine-grain $\mathrm{Nb}$ sheets involves many steps, including forging, rolling and heat treatments, to break the large crystals of the $\mathrm{Nb}$ ingot, which had been electron beam melted multiple times, into uniform fine-grains.

The idea of using ingot material for cavity fabrication in the form of sheets sliced directly from an ingot and shaping the slices with the standard deep drawing technology, rather than carving out the half cells from thick chunks of material as was done for the separator cavities at KfK, was introduced at Jefferson Lab in 2004. It turned out that this sliced material had very good mechanical properties and could be easily formed into half cells despite the large grains (the size of the grains is a few $\mathrm{cm}^{2}$ ). This review article includes the summary of several studies on material characterization (Sec. 2), cavity fabrication (Sec. 3) and testing (Sec. 4) around the world based on single crystal and large grain niobium. In Section 5, we will discuss the achievements and some open issues related to ingot $\mathrm{Nb}$ technology; a brief summary will be given in Sec. 6. Additional information on the topics discussed in this article can be found in several conference proceedings on SRF technology [10] and niobium materials for more details [11, 12].

\section{MATERIAL STUDIES}

Once it was determined that ingot slices could be used for SRF cavity fabrication, it became obvious that this choice of material could offer certain advantages over standard fine-grain material:

- Reduced cost because of elimination of sheet processing steps.

- Possibility of higher $Q_{0}$-values/lower residual resistance because of less grain boundaries.

- Statistical smooth surface can be achieved with buffered chemical polishing (BCP) only instead of electropolishing (EP) because of reduced number of grains.

- Reduced degradation of $Q_{0}$ due to thermal feedback with increasing RF field, because of a phonon peak in thermal conductivity.

- Reduced opportunity to introduce foreign impurities in the material, because of elimination of sheet processing steps.

However, there are some potential issues connected with this material: 
- Can one develop an effective/inexpensive slicing method?

- How uniform is the forming process, is slippage at grain boundaries problematic?

- Do grain boundaries cause a problem during welding?

- Are grain boundaries leak tight?

- Are different grain orientations reacting differently to chemical etching?

- Is oxidation behavior depending on grain orientation?

- Is field emission grain boundary dependent?

Many of the issues listed above are technological and provided a rich field of research for material scientists and surface scientists. Table 1 summarizes the different areas of research which have been addressed in the last ten years. Some of the results from this research are discussed in more details in the following sections.

Table 1: A summary of the material studies conducted on ingot $\mathrm{Nb}$ in recent years.

\begin{tabular}{|c|c|c|}
\hline Subject & Comments & References \\
\hline Mechanical & Yield strength, tensile strength, elongation & {$[13,14,15,16$} \\
\hline Properties & $\begin{array}{l}\text { for different grain orientation, bulging, } \\
\text { residual strain, formability }\end{array}$ & $17,18]$ \\
\hline Thermal properties & $\begin{array}{l}\text { Thermal conductivity, phonon peak, effect } \\
\text { of annealing and impurities, effect of strain }\end{array}$ & {$[13,19,20,21]$} \\
\hline Magnetic properties & $\begin{array}{l}H_{c 1}, \quad H_{c 2}, \quad H_{c 3} \text { for different crystal } \\
\text { orientations, temperature dependences, } \\
\text { penetration depth }\end{array}$ & $\begin{array}{c}{[20,22,23,24,} \\
25]\end{array}$ \\
\hline $\begin{array}{l}\text { Crystal orientation, } \\
\text { recrystallization }\end{array}$ & $\begin{array}{l}\text { Grain orientation in different materials, } \\
\text { dislocation density, dependence of etch rate } \\
\text { on orientation and residual strain }\end{array}$ & {$[26$} \\
\hline Flux penetration & $\begin{array}{l}\text { Magneto-optical investigations, influence of } \\
\text { grain boundaries on flux penetration }\end{array}$ & $\begin{array}{l}{[23,24,28,29} \\
30,31,32,33]\end{array}$ \\
\hline Oxidation / Hydrides & $\begin{array}{l}\text { Oxide composition for different crystal } \\
\text { orientations, sealing of surface, hydrides }\end{array}$ & {$[34,35]$} \\
\hline Field emission & $\begin{array}{l}\text { Emitter density, grain boundary segregation, } \\
\text { cleaning }\end{array}$ & {$[36,37,38]$} \\
\hline Fabrication & $\begin{array}{l}\text { Forming issues, welding, enlargement of } \\
\text { single crystal }\end{array}$ & {$[16,39]$} \\
\hline Surface topography & $\begin{array}{l}\text { Influence of polishing conditions, replica } \\
\text { technique, pits, roughness, field } \\
\text { enhancement }\end{array}$ & $\begin{array}{l}{[40,41} \\
42,43]\end{array}$ \\
\hline Hot spots, cold spots & $\begin{array}{l}\text { Point contact tunneling, vacancies, } \\
\text { dislocations }\end{array}$ & $\begin{array}{c}{[44,45,46,47} \\
48]\end{array}$ \\
\hline Surface impurities & Hydrogen depth profile & {$[49,50,51,52]$} \\
\hline
\end{tabular}

\subsection{Mechanical and Thermal Properties}

The ingot material originally provided by CBMM, as part of the cooperative research and development agreement had excellent uni-axial mechanical properties as shown in Fig. 2 for a single crystal piece cut from an ingot compared to the polycrystalline niobium. Figure 2(b) shows the stress-strain curves for different samples with different crystal orientations from a sheet of ingot material. 

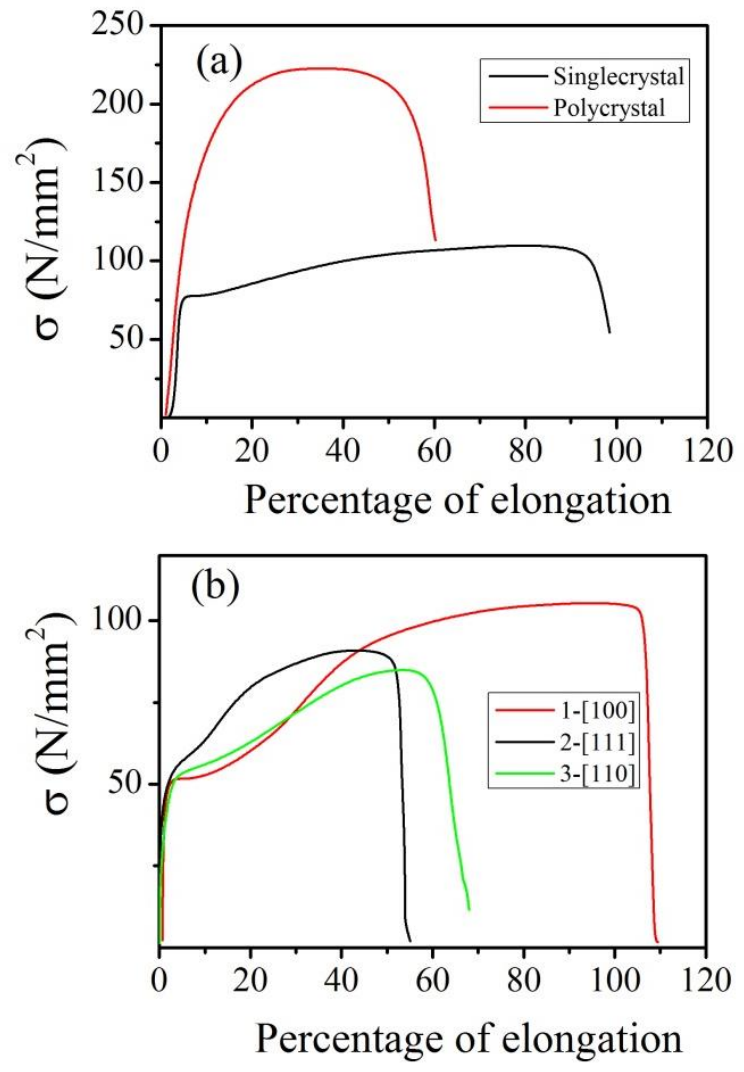

Fig. 2. (a) Stress-strain behavior of poly-crystal and single-crystal niobium. (b) Stressstrain curves for three different samples with different crystal orientations [100], [111], and [110] from a sheet of ingot material [16]. In all cases the elongation is sufficient for deep drawing of half-cells.

As part of the development program at CBMM for high purity niobium ingots, several ingots have been produced with different parameters during the melting process. The mechanical properties of samples cut from ingots with different Tantalum content and melting cycles showed a decreasing yield strength with increasing number of melts, as expected due to the increased purity of the material [53].

More stringent testing of the material properties relevant to forming into cavity cells and conforming to the pressure vessel code is done with a bi-axial bulging test. Such testing was done at DESY: the percentage elongation to fracture is low $(<15 \%)$ and the rupture takes place close to a grain boundary. Although this might have some impact on the formability of large-grain niobium sheets, rupture at grain boundaries was not encountered in the fabrication of cavities from this material.

The thermal conductivity of both single crystal and large grain niobium was measured at several labs and Fig. 3 shows results obtained at Jefferson lab [13], DESY [16] and MSU [19]. At MSU the thermal behavior of large grain material was further investigated with respect to the dependence of the phonon peak on mechanical deformation, uptake of hydrogen and different RRR values. As it has been reported in 
[21], the phonon peak is very sensitive to mechanical crystal strain, but can be recovered by annealing heat treatments. The magnitude of decrease of the phonon peak is not only related to the degree of deformation, but is also strongly influenced by the grain orientation along the direction of deformation, because of the different number of slip systems being activated during deformation. The results showed that a $1000{ }^{\circ} \mathrm{C}$ heat treatment in vacuum for $2 \mathrm{~h}$ is sufficient to recover the phonon peak in grains for which it was reduced by up to $74 \%$ by strain-induced dislocations [21]. Hydrogen in niobium also affects the thermal conductivity especially in the phonon peak region; however a full recovery can be achieved after degassing at $800{ }^{\circ} \mathrm{C}$ for $3 \mathrm{hrs}$. The phonon peak height also increases with increasing RRR value and it is not affected by the presence of a single grain boundary (GB) [21].

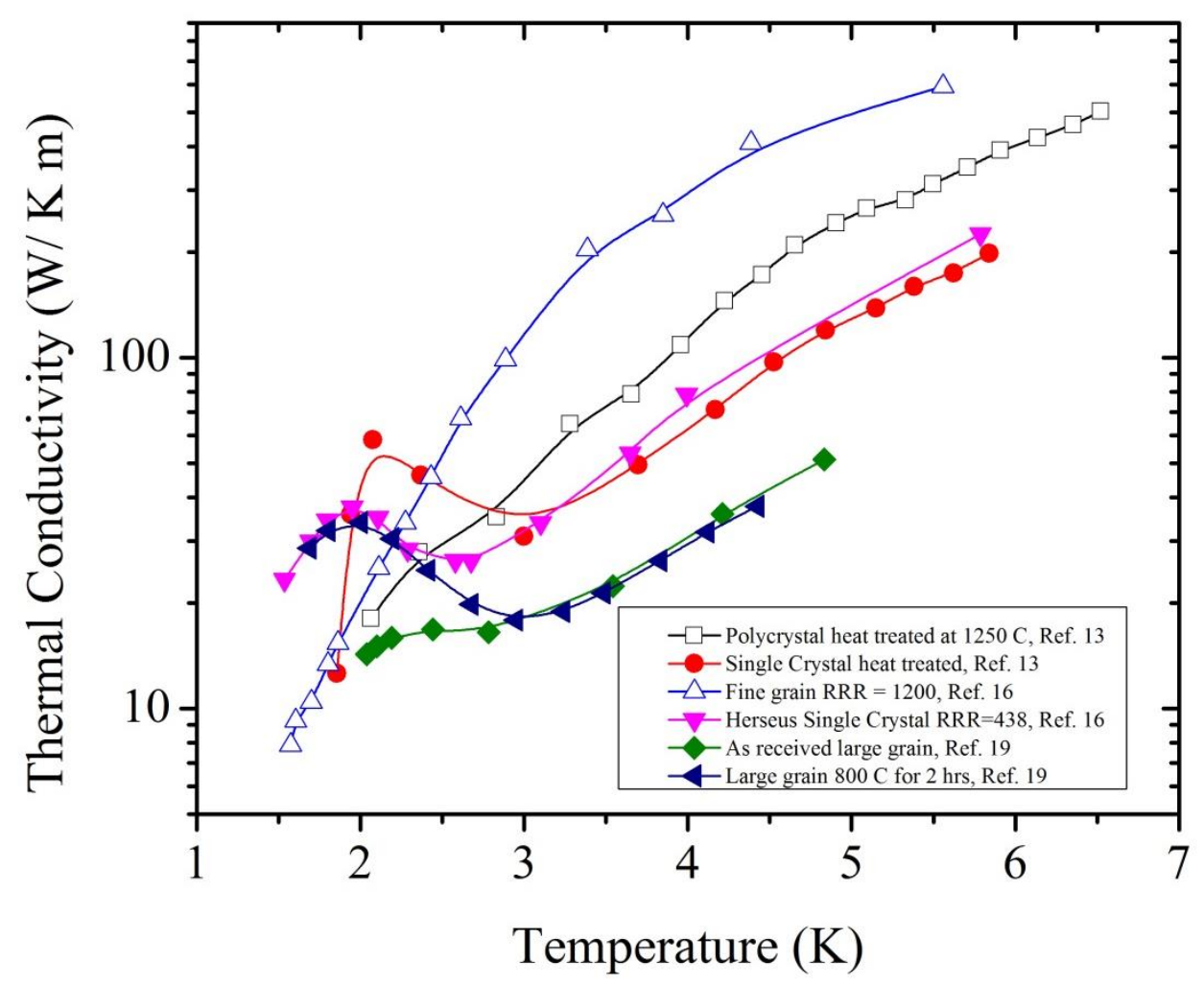

Fig. 3. Thermal conductivity of single-crystal and large-grain ingot niobium compared to fine-grain $\mathrm{Nb}$. A phonon peak is typically measured in ingot niobium, compared to finegrain material.

\subsection{Formability, Crystal Deformation and Crystal Structure}

For material scientists it is of great interest to understand how the crystal structure is responding to severe deformations such as those applied during deep drawing of cavity half-cells. During the deep drawing process a plastic strain differential is created through the material, which induces residual stresses. Their magnitude depends on yield stress, the accumulated plastic strain and the hardening of the material, all influencing the formability of the material and spring back behavior after forming. First measurements of 
residual stresses and formability of large grain and single crystal niobium were reported in [15]. Because of the large strain reserve for uni-axial testing and an estimated maximum local strain of $\sim 20 \%$ there is a large margin even for the biaxial nature of the forming. However, anisotropy in yielding provides a potential for local thinning and shape deviations. This has been observed at many places, especially slippages at grain boundaries.

Microstructure, dislocation density, texture and slip behavior of ingot $\mathrm{Nb}$ samples have been studied at MSU aiming at providing information needed for computational simulation of the forming processes in order to predict deviations of half-cells from the desired shape [17]. A Laue camera method was also developed to measure the crystal orientation of $\sim 26 \mathrm{~cm}$ diameter discs and the analysis of discs from different ingot manufacturers showed no commonalities in terms of preferred crystal orientations, suggesting that the ingot solidification process is controlled by random processes [26].

\subsection{Surface impurities, Oxides and Hydrides}

Sheets from ingot material usually consist of several large grains with decreasing grain size towards the outer circumference (this depends on the size of the water-cooled crucible during the melting process). These grains have typically different crystal orientations and react differently to mechanical deformations, to chemical agents such as BCP acid mixture and show also different oxidation behaviors. Several investigations as discussed below have addressed these issues. Single crystal niobium samples of (100), (110), and (111) crystal orientations were analyzed using TEM and SIMS techniques [34]. For all three orientations the uniform oxide layer thickness ranges from $4.9 \mathrm{~nm}$ for (100) orientation to $8.3 \mathrm{~nm}$ for (110) orientation (only one sample of each orientation was analyzed) and no significant sub-oxides were seen. The oxide thickness measured on a fine-grain sample by TEM was $\sim 8.2 \mathrm{~nm}$ with a sharp metal/oxide interface [54]. SIMS measurements were made on samples of all 3 orientations to obtain depth profiles of hydrogen, carbon and oxygen after heat treatments at $90^{\circ} \mathrm{C}, 600^{\circ} \mathrm{C}$ and $1250{ }^{\circ} \mathrm{C}$. High levels of hydrogen were found between the oxide layer and the niobium, but low levels in the oxide. Below the oxide layer no high oxygen concentration regions were detected and carbon contamination was mainly located on the surface.

Hydrogen depth profiles have been measured on fine-grain and large-grain samples using different techniques. Measurements by SIMS on samples after hightemperature $\left(>600{ }^{\circ} \mathrm{C}\right)$ vacuum annealing showed a large reduction of $\mathrm{H}^{-} / \mathrm{Nb}^{-}$after annealing and higher $\mathrm{H}^{-} / \mathrm{Nb}^{-}$for fine-grain samples compared to large-grain ones after similar heat treatment [44]. Hydrogen depth profiles of fine-grain $\mathrm{Nb}$ samples were measured by Nuclear Reaction Analysis using a $6.4 \mathrm{MeV}{ }^{15} \mathrm{~N}$ beam and the results showed a decrease of the sub-surface $\mathrm{H}$-peak after vacuum annealing at $800{ }^{\circ} \mathrm{C}$ and after low-temperature (100-140 ${ }^{\circ} \mathrm{C}$ ) baking (LTB) in ultra-high vacuum $[55,56]$. Elastic recoil detection analysis (ERDA) using $1.6 \mathrm{MeV}^{4} \mathrm{He}$ beam had also been used for hydrogen depth profiling of fine-grain and large-grain samples after chemical treatments, hightemperature vacuum annealing and LTB. Even though in all samples after different treatments hydrogen peaks were detected in a near surface sheath of $\sim 8 \mathrm{~nm}$, of the order of the depth resolution, no difference in hydrogen depth profile was seen after LTB or between samples cut from cavities at locations of high and low rf losses (referred to as "hotspots" or "coldspots") [47]. 
Raman spectroscopy was recently applied to study the contamination of largegrain samples within a $\sim 10-20 \mathrm{~nm}$ depth from the surface [52]. The results were consistent with the presence of hydrocarbons and hydrides in samples which had "etch pits" associated with enhanced rf losses. Direct observations of the formation of hydrides on the surface of fine-grain and single-crystal niobium samples are reported in [35] but the influence of material structure on hydride precipitation could not be clearly studied because of the much higher initial $\mathrm{H}$ concentration in the single-crystal samples, due to the preparation methods.

Hydrogen is a notoriously difficult element to study in $\mathrm{Nb}$ and only few techniques are available to measure its depth profile in $\mathrm{Nb}$. Hydrogen is a well-known cause of increased losses in $\mathrm{Nb}$ cavities due to hydride formation (known as "Q-disease") and a similar mechanism was recently proposed to explain the occurrence of anomalous losses above $\sim 90 \mathrm{mT}$ (known as "Q-drop" or "high-field Q-slope") [57]. More extended and systematic studies on a large number of carefully prepared samples are needed to gain a better understanding of sub-surface $\mathrm{H}$ in $\mathrm{Nb}$ and whether differences due to the material structure (fine-grain vs. large-grain) influence the amount and mobility of $\mathrm{H}$ in $\mathrm{Nb}$. Characterizing the same samples using multiple techniques would be particularly useful to help reconcile some of the discrepant results in the literature and highlight some of the limitations of each technique.

\subsection{Chemical Etching and Surface Finish}

Superconducting niobium cavities typically undergo a surface treatment to remove the "surface damage" layer after fabrication. It has been shown in series of tests with poly-crystalline niobium treated by BCP that the "damage layer" is of the order of 100-150 $\mu \mathrm{m}$ thick [58]. The crystallographic structure however is already restored after $\sim 10 \mu \mathrm{m}$ material removal. Other methods used in the preparation of rf cavities are EP and/or centrifugal barrel polishing (CBP) and for very high accelerating gradients these methods have been superior to BCP. A study on the performance of a $1.5 \mathrm{GHz}$ single-cell cavity made of ingot $\mathrm{Nb}$ for increasing amount of material removal indicates the possibility of achieving peak surface magnetic field of $\sim 120 \mathrm{mT}$ at $2.0 \mathrm{~K}$ after $\sim 70-100$ $\mu \mathrm{m}$ removal by BCP only [59], as shown in Fig. 4. 

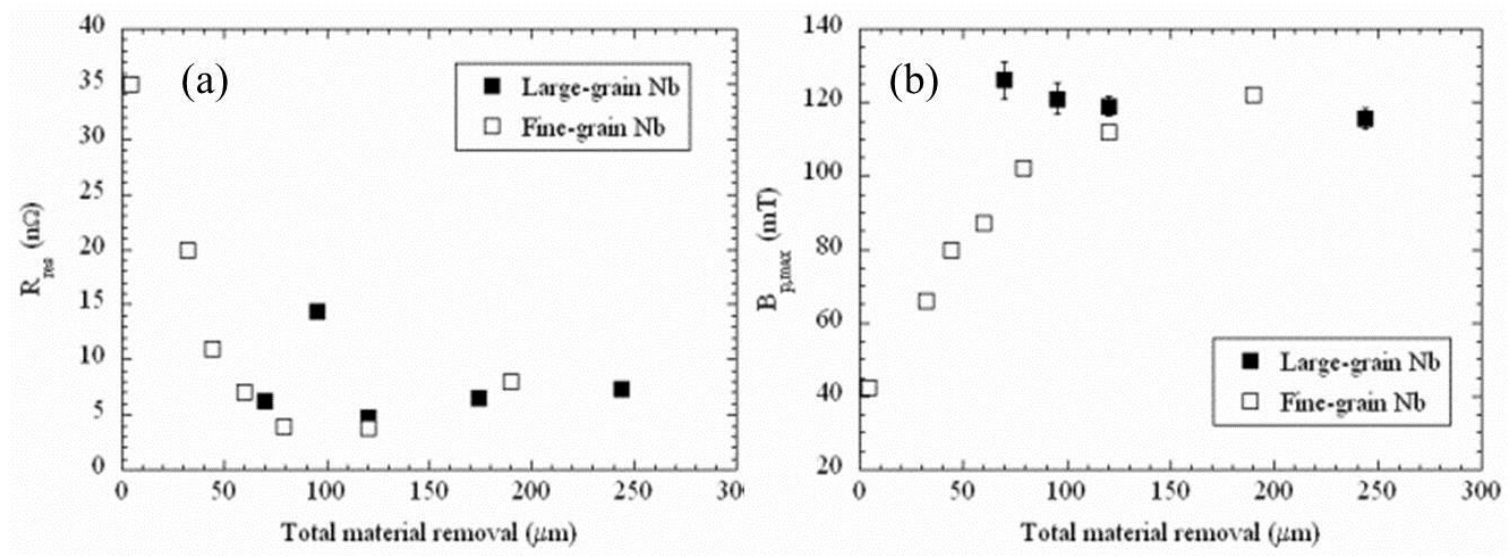

Fig. 4. Dependence of the residual resistance (a) and maximum peak surface magnetic field at $2.0 \mathrm{~K}$ (b) on material removal by $\mathrm{BCP}$ for a fine-grain [58] and ingot $\mathrm{Nb} 1.5 \mathrm{GHz}$ cavity [59].

Since one of the expectations for large grain material is less expensive preparation procedures, some emphasis has been placed on the achievable surface finishes with different methods. Roughness measurements by atomic force microscopy (AFM) over a $(80 \times 80) \mu \mathrm{m}^{2}$ area of large-grain sample with different orientations showed a root-meansquare (RMS) roughness of $\sim 70-110 \mathrm{~nm}$ after $30 \mu \mathrm{m}$ BCP, decreasing to $\sim 7-9 \mathrm{~nm}$ after removal of $\sim 100 \mu \mathrm{m}$. Typical RMS roughness measured by AFM over a $(100 \times 100) \mu^{2}$ area of fine-grain samples after $100 \mu \mathrm{m}$ removal by BCP and after $50 \mu \mathrm{m}$ removal by EP are $\sim 460 \mathrm{~nm}$ and $\sim 70 \mathrm{~nm}$, respectively [40,41].

However, besides the excellent intra-grain smoothness, differential etching of different crystal orientations by BCP cause the occurrence of tens of micrometers high "steps" at grain boundaries. Mechanical buffing of grain boundary steps, either with hand tools on half-cells, or with centrifugal barrel polishing (CBP) on the entire cavity surface have been applied in some cases. Nevertheless, work on 9-cell cavities described in Sec. 3 did not find that the presence of grain boundary steps limits the cavity performance.

\subsection{Grain Boundary Losses}

Some of the weak areas in a cavity surface are the grain boundaries, which are chains of dislocations, where flux can enter preferentially. Using magneto-optical imaging (MO) it was shown on fine grain niobium $[28,29]$ that some grain boundaries indeed showed preferential flux penetration at a field lower than that for which flux penetration occurs within the grains, the first experimental evidence of depressed superconductivity at a grain boundary in $\mathrm{Nb}$. This study had left some questions open to what extent did surface topology and chemical surface treatments, as well as heat treatments to enlarge the grain size, influence the flux penetration behavior. Large grain/single crystal niobium provided the opportunity for "cleaner" conditions to investigate the mechanism of flux entry at a grain boundary and flux flow along a grain boundary. Such investigations have been carried out on bi-crystal and tri-crystal large grain niobium samples [30]: in a bi-crystal with $<1 \mu \mathrm{m}$ step at the grain boundary and the grain boundary plane nearly parallel to the applied magnetic field, preferential flux entry was observed at field levels far below $H_{c l}$, whereas at large steps and/or grain 
boundaries not parallel to the field no preferential penetration was observed, excluding topological features causing this behavior. A continuation of this work showed that flux penetration will also occur at the "stable" boundary, if it is rotated such that it is parallel to the applied field. TEM images suggest that dense dislocation arrays at the sample surface possibly introduced by the preparation techniques such as cutting and grinding, are responsible for the reduced $H_{c l}$.

The presence of impurities, oxides or hydrides at grain boundaries, introduced during surface treatments, can locally depress the superconducting energy gap, reducing the depairing current density and enhancing the flux flow resistivity. A detailed study showed evidence of preferential flux flow when the external magnetic field is greater than $\sim 80 \mathrm{mT}$ and the dissipation is highest when the field is parallel to the plane of the grain boundary [23]. The surface treatment of the bi-crystal sample also had an effect, with EP resulting in less-pronounced flux flow above $\sim 100 \mathrm{mT}$, compared to BCP treatment.

In the case of ingot material the density of grain boundaries is lower, but the length of these grain boundaries is also longer, therefore it is difficult to assess its advantage compared to fine-grain niobium, especially since the grain boundaries in formed cavity cells are randomly oriented with respect to the rf field. To further evaluate the impact of grain boundaries on the performance of SRF Nb cavities, a series of experiments were conducted with cavities made from material with different grain sizes. Four $2.2 \mathrm{GHz}$ cavities of identical shape made from high purity niobium with single crystals, large grains of the order $\mathrm{cm}^{2}$ size (2 cavities) and standard polycrystalline niobium were investigated [60]. After several steps of identical procedures (for material removal only BCP was used) the cavities showed very similar behavior after a final step of post-purification and LTB with a slightly higher quench field $(\sim 150 \mathrm{mT}$ at $2.0 \mathrm{~K})$ for the single crystal material. This result does not point to any significant influence of grain boundaries in limiting the performance of these cavities.

\subsection{Magnetic Properties}

In the early 1970's a program was started at KFZ Karlsruhe to develop new methods to investigate "SRF grade" niobium using the conduction electrons as a probe to sample material properties at different depth in the material. Volume properties such as residual resistivity and bulk mean free path were investigated with dc and $10 \mathrm{kHz}$ penetration depth measurements. Measurements of flux pinning and magnetization $\left(H_{c 2}\right)$ explore depths between 1 and $50 \mu \mathrm{m}$, whereas penetration depth measurements as a function of a magnetic field parallel to the niobium surface probe a depth of 0.04 to $1 \mu \mathrm{m}$ and are useful to determine surface mean free path and sub-oxide precipitates. The niobium/oxide interface can be sampled with $H_{c 3}$ and tunnelling measurements to a depth of $\sim 40 \mathrm{~nm}$ [61]. This type of measurements have been recognized in recent years as a powerful method to gain information about surfaces subjected to different cavity treatments such as BCP, EP, heat treatment, and "in-situ" baking [20, 22, 24].

Magnetization measurements give information about bulk properties such as magnetic field for first flux penetration $\left(H_{f f p}\right), H_{c 2}$ and irreversibility, related to pinning centers in the material. Magnetization measurements were carried out on $120 \mathrm{~mm}$ long large grain cylindrical rods of different RRR-values using the setup described in Ref. [24]. The results showed that surface and heat treatment have little influence on the first 
flux penetration field $\left(H_{f f p} \sim 170-190 \mathrm{mT}\right)$, whereas the hysteresis is significantly reduced after the $800{ }^{\circ} \mathrm{C}$ heat treatment. Furthermore, the critical current density and pinning force calculated from magnetization data indicate that LG samples have lower critical current density and pinning force density compared to FG samples, therefore favoring lower flux trapping efficiency [24]. This effect may explain the lower values of residual resistance often observed in LG cavities than FG SRF cavities [62].

Isothermal magnetization curves at $2.0 \mathrm{~K}$ for fine-grain and large-grain samples, 2 $\times 2 \times 2 \mathrm{~mm}^{3}$ in size, were measured after cutting, BCP and vacuum annealing [25] and the field of first flux entry was obtained from the data using the method discussed in [63]. The results showed a delayed flux entry up to $180 \mathrm{mT}$ in large-grain pristine samples whereas $H_{\text {ffp }}$ is reduced to $\sim 100 \mathrm{mT}$ after BCP but increased to $\sim 120 \mathrm{mT}$ after annealing. The samples display a large hysteresis loop and several flux jumps occurred after cutting. After BCP and heat treatment, the hysteresis is significantly reduced and flux jumps are absent. This result is consistent with a reduction of pinning centers in the surface after $\mathrm{BCP}$ as well as heat treatment. The results on fine-grain samples are similar to those for the large-grain ones with $H_{\text {ffp }}$ lower by $\sim 10 \%$. The causes for differences on the dependence of $H_{\mathrm{ffp}}$ on surface treatments measured on the small, cube-shaped samples in Ref. [25] versus that obtained from cylindrical rod-shaped samples in Ref. [24] are not clear. This might be due to different instrument sensitivities or to the different surface-tovolume ratio between the two types of samples.

Measurements of the change in penetration depth as a function of temperature give information about the mean free path in the probed layer $(\sim 25 \mu \mathrm{m}$ at $300 \mathrm{kHz})$. It is a sensitive measurement of the magnetic properties in the surface layer up to $H_{c 3}$. These investigations were carried out by measuring the frequency shift of a $300 \mathrm{kHz}$ LC oscillator, in which the coil - the inductance of the circuit - was tightly "filled" with the sample under test. The change in the LC-oscillator's resonance frequency, which is proportional to the change in penetration depth, is measured as the function of external magnetic field [24]. The hysteresis was observed between $H_{\mathrm{ffp}}$ and $H_{\mathrm{c} 2}$ is related to surface pinning and the ratio of critical fields $H_{\mathrm{c} 3} / H_{\mathrm{c} 2}$ at increases up to $\sim 1.98$ after LTB consistent with earlier results [22]. $H_{\text {ffp }}$ is $\sim 170 \mathrm{mT}$ in both large-grain and fine-grain samples and did not change significantly after LTB.

A set of experiments conducted at Helmholtz Zentrum Berlin (HZB) investigated the flux trapping and flux release properties of fine-grain, large-grain and single-crystal niobium subjected to different surface treatments [32,33]. Magnetic flux could be trapped in the disc or rod-shaped samples by applying an external magnetic field during cool down below $T_{c}$ and the amount of trapping was monitored with a flux gate magnetometer. The samples were then heated and the flux released could again be measured with the flux gate magnetometer. The experiments showed that $100 \%$ of flux was trapped in the fine-grain material, independently on cooling rate, whereas in the single-crystal material annealed at $800{ }^{\circ} \mathrm{C}$, the flux trapping depended logarithmically on cooling rate and saturated roughly between $60-70 \%$. Since the mobility of flux lines is temperature dependent and increases towards lower temperature, the measurements indicate a significantly smaller pinning force in the annealed single crystal material, consistent with results obtained from magnetization measurements at Jefferson lab [24]. 


\subsection{Tunneling Measurements}

Tunneling experiments can be used to investigate the electronic structure and density of states of niobium surfaces after different surface treatments. The reason for such investigations would be to clarify the importance of the niobium-oxide interface in causing the non-linearities in $Q_{0}$ vs $E_{a c c}$ tests as suggested, for example, by the "interface tunnel exchange" model of ref. [64]. The instrumentation for the point contact tunneling consists of a gold tip touching the niobium sample under test and the I-V curve and its derivatives $d I(V) / d V$ are taken. Such a measurement was done on an electropolished single crystal sample after air exposure and after low temperature baking in air at $120{ }^{\circ} \mathrm{C}$ for $48 \mathrm{hrs}$. The conductance measurement gives a gap parameter of $\Delta=1.55 \mathrm{meV}$ indicative of bulk niobium, but also shows a smearing of the density of states, which was attributed to magnetic scattering caused by the magnetic moments of the nonstoichiometric surface oxides on the niobium. After baking, the broadening is somewhat reduced, hinting at the formation of a more stoichiometric oxide with reduced magnetic moments [45]. In Ref. [65] it is mentioned that similar results were obtained for finegrain samples.

The point contact tunneling method was successfully used to find differences between so-called "hot" spot samples and "cold" spot samples cut out from large-grain niobium cavities [45], which had been tested with temperature mapping and had shown different power dissipation patterns on the cavity surface. The analysis of these measurements showed that "hot" samples have a lower energy gap $(1.2 \mathrm{meV}<\Delta<1.55$ $\mathrm{meV}$ versus $1.5 \mathrm{meV}<\Delta<1.55 \mathrm{meV}$ for "cold" samples) and a larger pair-breaking parameter as compared to the "cold" samples. In addition spectra with a large zero-bias conductance peak were measured on "hot" samples. The results are indicative of a larger fraction of unpaired electrons and the presence of magnetic impurities near the surface, causing increased dissipation in RF fields.

\subsection{Field Emission}

Enhanced field emission is one of the limiting phenomena in niobium SRF cavities. At high peak electric RF fields $\left(E_{\text {peak }} \geq 40 \mathrm{MV} / \mathrm{m}\right.$ ) electrons are drawn out of the niobium surfaces or contaminating particulates and are accelerated by RF field inside the cavity. When impacting on opposing surfaces, they generate X-rays and additional heating of the surface, degrading the performance of the cavities. First experiments on niobium samples were done at CERN in $~ 1985$ [66] in a DC test set-up to explore the nature of field emission sites and to correlate the results with field emission in RF cavities. Recent field emission studies at University of Wuppertal were focused on largegrain and single-crystal samples [37, 38]. The results indicated that roughness influences the field emission behavior, as rougher surfaces showed higher density of emitters at a given field and lower field emission onset, favoring etched single-crystal surfaces over those of large-grain or fine-grain samples. No field emission from grain boundaries was detected up to electric fields of $250 \mathrm{MV} / \mathrm{m}$. Heat treatments in ultra-high vacuum also affect the field emission behavior of $\mathrm{Nb}$ surfaces, as an increasing number of emitters are activated with increasing heat treatment temperature. 


\section{CAVITY FABRICATION}

\subsection{Slicing of ingot $\mathrm{Nb}$}

Besides the aspects of material formability, crystal structure and crystal deformation there is the technical aspect of fabricating cavities from ingot material in a reliable and cost effective way. This includes the melting of the ingot, the slicing to sheets, deep drawing and reproducibility of deep drawn parts, machining and electron beam welding. One of the first large grain ingots Jefferson lab received from CBMM had a very large single crystal in the center of the ingot as shown in Fig. 5(c). This is a very desirable configuration - for deep drawing of half cells the highest deformation occurs in the center and a single crystal in this area provides uniform mechanical properties however, more often the ingot consists of several large grains. Unfortunately it was unknown under which conditions and configuration the ingot from which the slice shown in Fig. 5(c) was grown and how it could be reproduced.

Several attempts have been made to grow single crystal ingots. Within the framework of an R\&D program with DESY, W.C. Heraeus investigated the growth dynamic [67] in an attempt to produce reliably ingots with a large central grain and a specified orientation as required by the DESY specifications [68]. There are many variables in Electron Drip Melting, which is the only applicable process for industrial use (schematically shown in Fig. 6), influencing the nucleation and dissolution of grains in the liquid pool such as local pool temperature, molten pool motion, external vibrations, dripping of melt-stock into the pool, vibration from the withdrawal of the ingot [69].
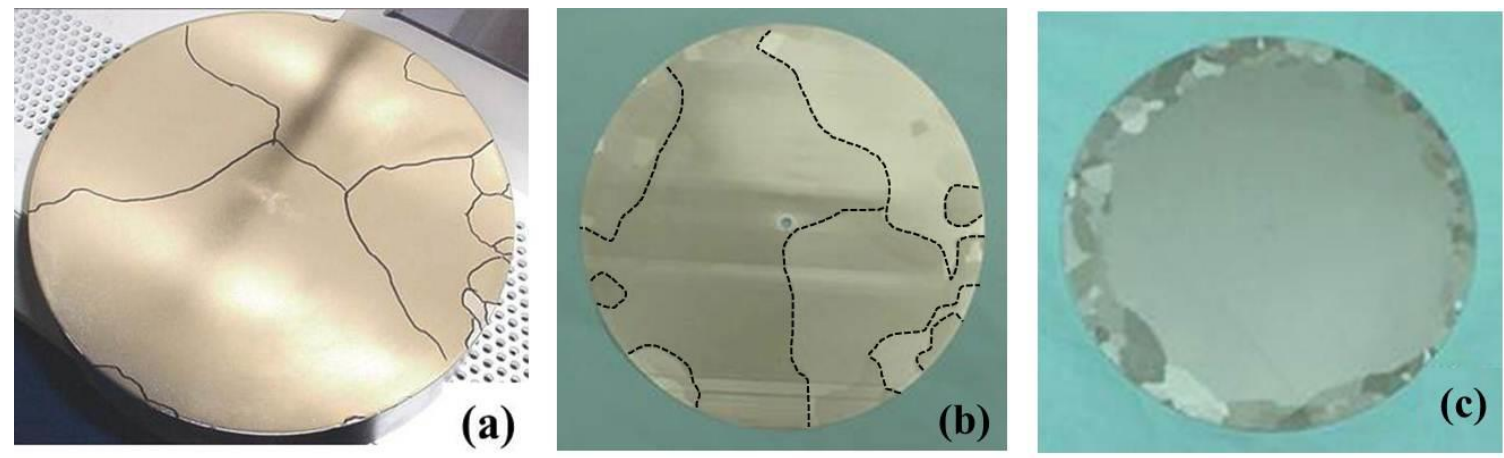

Fig. 5. Large grain ingots from CBMM; (a) and (b) have a more typical grain structure, (c) has a large single crystal at the center. Grain boundaries are highlighted in (a) and (b) with a black marker. 


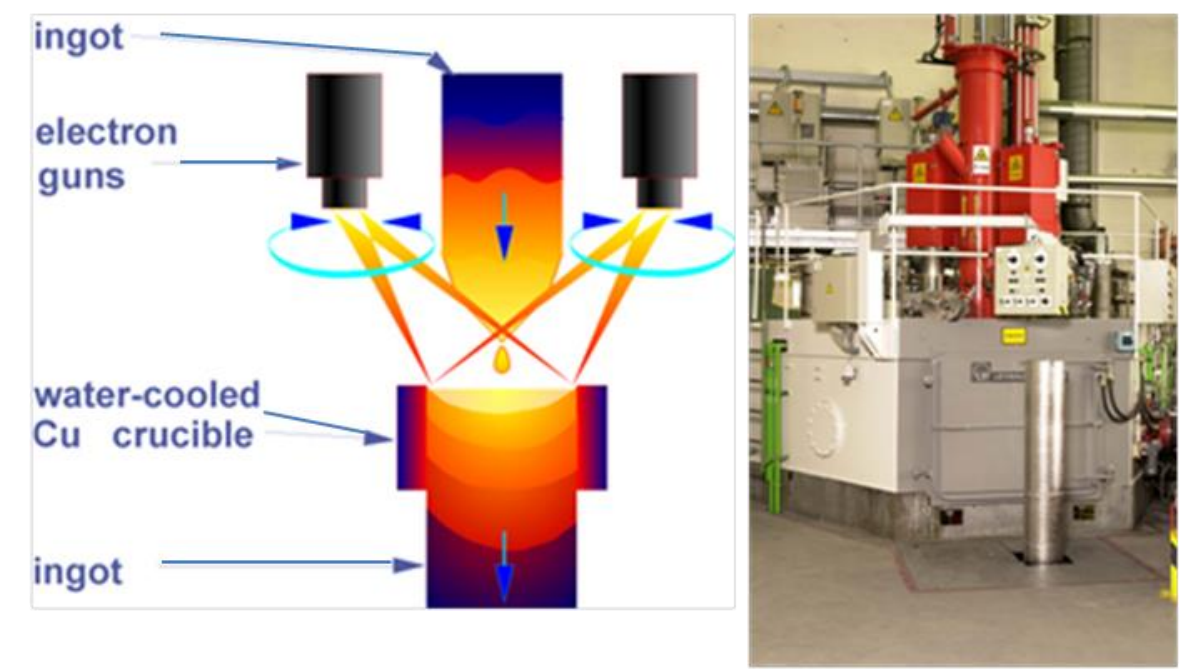

Fig. 6. Schematic of drip melting in an Electron beam melting furnace (left) and furnace at W.C. Heraeus (right).

The efforts at W.C Heraeus included variation of melting/cooling parameters, such as modification of the beam shape, position and energy entry and cooling of the crucible side wall or bottom. These efforts resulted in the realization that the melting process is not sufficiently stable to reproducibly create a central crystal of $150 \mathrm{~mm}$ diameter with a specified crystal orientation throughout a whole ingot of approximately $2000 \mathrm{~mm}$ length. However, the company succeeded in providing 250 large grain discs to DESY from several ingots. Further efforts in growing single crystal ingots have been reported in ref. [70]; at Tokyo Denkai (TD) a seed crystal specimen was placed on a niobium bottom plate and the standard melting process was started. Ultrasonic tomography showed that the seed crystal was not sufficiently thick and that the base plate crystals determined the crystal growth in the ingot. It is concluded that a thicker seed crystal is needed for a successful single crystal growth.

A cost-effective slicing process was developed at W.C. Heraeus to provide sheets for deep drawing of cavity half cells from an ingot. The slicing procedure used the same multi-wire technique as it is used for silicon wafer slicing. This method was subsequently further developed at KEK involving Japanese industry for "mass production". Presently a powerful multi-wire slicing machine using very thin piano wire $(0.16 \mathrm{~mm})$ and a slurry of abrasive material continuously applied to the wires is installed at Tokyo Denkai with a capacity of slicing simultaneously 150 discs from an ingot of approximately $28 \mathrm{~cm}$ diameter in about $48 \mathrm{~h}$. A thickness tolerance of $\pm 0.1 \mathrm{~mm}$ and average surface roughness of 0.3-0.6 $\mu \mathrm{m}$ as well as no degradation of the material purity were demonstrated with this technique [71], therefore satisfying the same requirements for standard fine-grain sheets. Figure 7 depicts the slicing schematic and actual arrangement. Eighteen discs for the fabrication of a $1.3 \mathrm{GHz}$ 9-cell cavity were checked by eddy-current scanning at DESY for imperfections and foreign inclusions with none of those being found. Therefore it was decided not to scan the remaining 144 discs required for the fabrication of additional 8 cavities. As mentioned in Sec. 4.2, the RF performance of those cavities was excellent, proving the high-quality of the material and of the cutting process. 


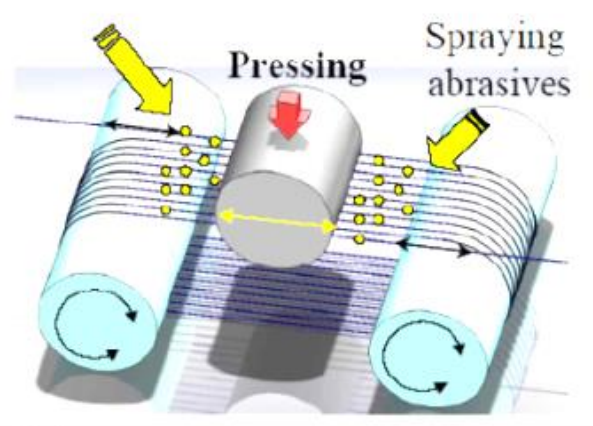

(a)

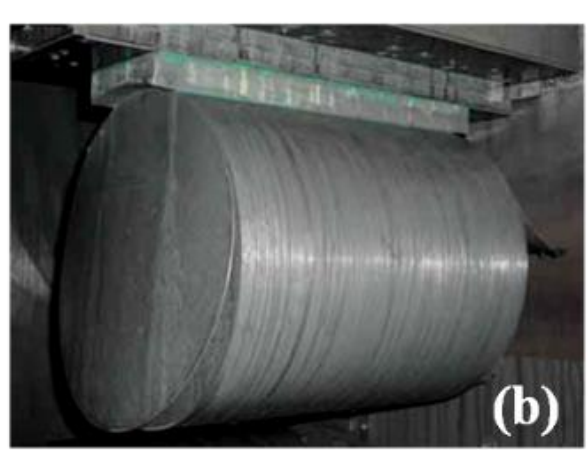

(b)

Fig. 7. (a) Schematic of multi-wire slicing, (b) actual slicing arrangement at TD [70].

\subsection{Material costs}

One of the expectations for the ingot material was the reduced material costs compared to sheet stock due to a much reduced handling and material waste caused by the rolling process. Figure 8 shows schematically the simplified production process [72]: instead of having 16 processes steps from starting material to final product in the conventional sheet production sequence, the number of handling procedures is reduced to 7. Additionally, the amount of material waste to produce ingot $\mathrm{Nb}$ discs was estimated to be $\sim 15 \%$, compared to $\sim 45 \%$ for standard fine-grain sheets [72]. The cutting of the ingot can still be relatively expensive, depending on how a company decides to amortize the cost of the slicing machine. Clearly, the ultimate cost of the material will depend on the specifications (for example the RRR and Ta contents), which can be different for different projects, the size of the market and the number of suppliers. Based on reduced processing steps and material waste, it is however reasonable to expect a lower cost of ingot material compared to fine-grain material. 


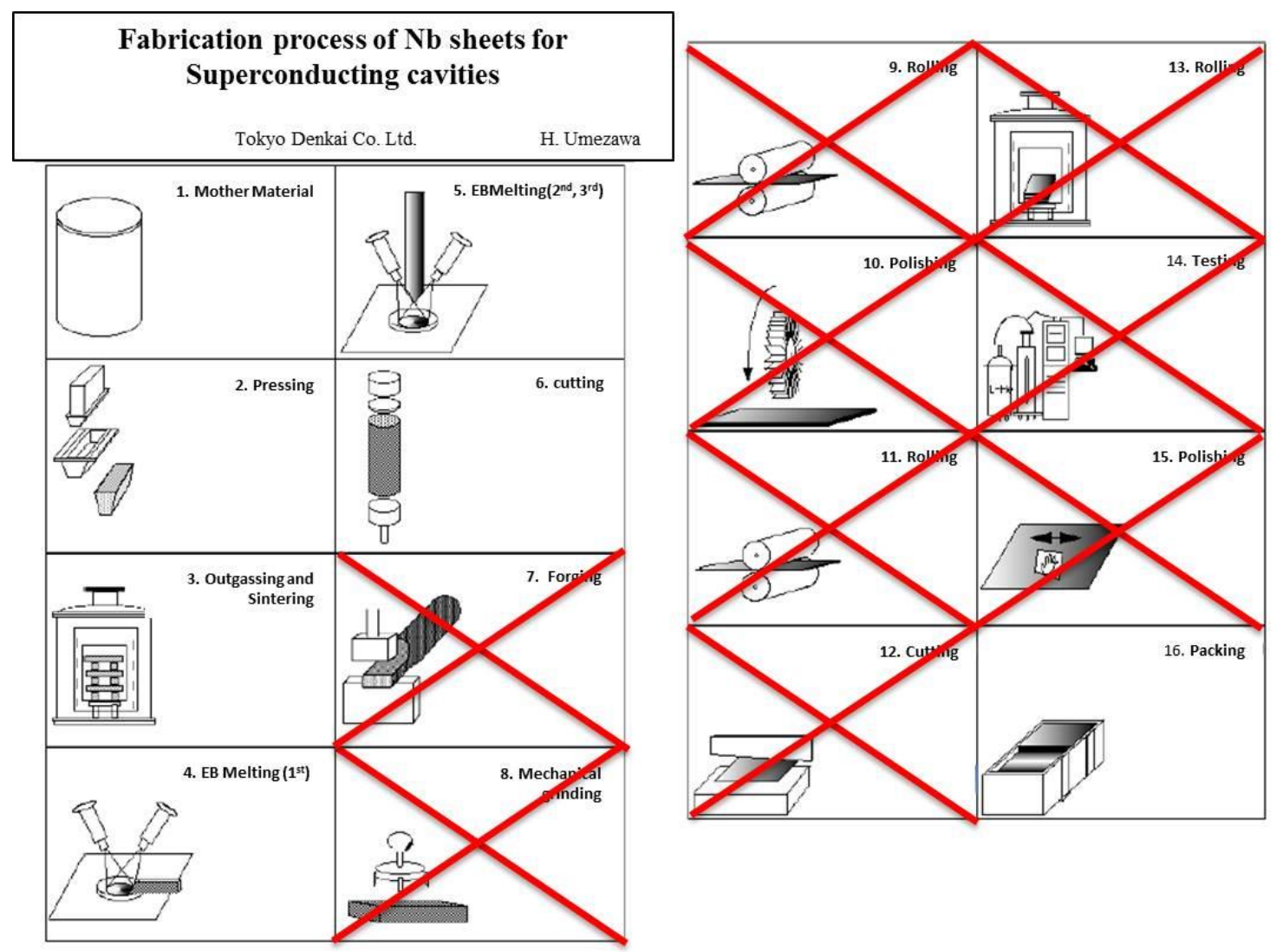

Fig. 8. Schematic of the production process for standard fine-grain sheets. Steps crossed in red are not required for producing ingot niobium sheets [72].

\subsection{Cavity Fabrication}

The first single cell $1500 \mathrm{MHz}$ cavity of the "High Gradient" (HG) shape [73], was fabricated in 2004 at Jefferson Lab from slices obtained by wire electro-discharge machining (EDM) from CBMM ingot niobium using the same fabrication techniques developed for fine-grain material. The half-cells show after deep-drawing ragged edges due to non-uniform deformation of the large crystals (Fig. 9). This is not an issue since, regardless of the discs' texture, extra-material is always left at both iris and equator and is later trimmed to achieve the desired length and frequency. In $\sim 2-3 \%$ of the cases, excessive thinning or ripping in the iris region when grain boundaries came together, as well as "oval" shaping due to different crystal orientations and slippage of grain at the grain boundaries occurred. In this respect, the presence of a large single-crystal at the center of the disc, as requested by DESY, would help mitigating these issues. Alternatively, discs with smaller grain size $\left(\sim 1 \times 1 \mathrm{~cm}^{2}\right)$ than those shown for example in Fig. 5 could be used as well.

Ingot discs which were saw-cut and machined to final thickness by Ningxia, China, failed during the deep-drawing process because of the high stresses introduced by saw-cutting and machining. Half-cells could be deep-drawn from this material after annealing the discs in a vacuum furnace at $600{ }^{\circ} \mathrm{C}$ for $10 \mathrm{~h}$. 

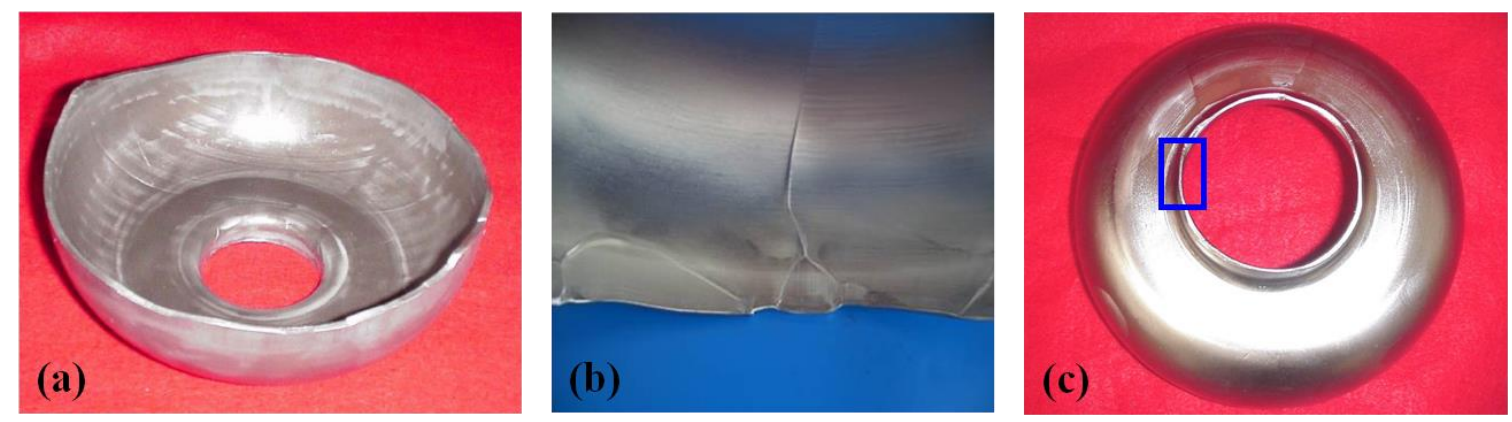

Fig. 9. A half-cell deep drawn from large-grain niobium discs at Jefferson Lab showing (a) earing, (b) grain boundary slippage and (c) thinning as indicated.

Electron-beam welding (EBW) of ingot Nb half-cells at Jefferson Lab was done using the same parameters used for EBW of fine-grain material. The deviation from roundness of ingot $\mathrm{Nb}$ half-cells was greater than for fine-grain ones and required more careful assembly procedure for EBW. More than fifty 1.3-1.5 GHz ingot Nb single-cells have been welded over the years with no issues. Ten 1.3-15 GHz multi-cell cavities from ingot $\mathrm{Nb}$ for different suppliers have been built so far at Jefferson Lab. Unfortunately, at least one hole during the equatorial EBW (usually the last one to complete the cavity) had to be repaired during the fabrication of multi-cell cavities and it was not clear whether the issue was related to the material. Defects in the equatorial EBW of large-grain cavities were rarely found after $\mathrm{CBP}$ and $\mathrm{EP}$ at KEK and inside-welds were adopted to eliminate the issue [74]. A recent study of EBW parameter was done at KEK, indicating that the same ones used for equator welds of fine-grain material can be used for welding largegrain [75]. A noticeable difference between EBW of large-grain Nb and that of fine-grain $\mathrm{Nb}$ is the absence of a "heat-affected zone" which has progressively smaller grains away from the weld approaching the $\sim 50 \mu \mathrm{m}$ typical grain size of the fine-grain material. Defects in this region had been found occasionally to be limiting the performance of SRF cavities made of standard fine-grain $\mathrm{Nb}[76,77]$.

As mentioned, Jefferson lab has fabricated $\sim 50$ single cell cavities from material of different suppliers and these cavities have been evaluated in more than 200 cryogenic RF tests. Additionally, four 9-cell cavities of the TESLA and ICHIRO [78] shape, two 7cell HG cavities, two 7-cell LL cavities, one 7-cell ILC/LL shape and one 5-cell "High Current" shape [79] cavity have been built. "Unconventional" cavity shapes such as a crab cavity, a 3.5 cell photo-injector cavity, several 0.5 cell cavities and 1.5 and 3.5 cell photo-injector cavities complete the Jefferson Lab "inventory". Figure 10 shows several of these cavities. 

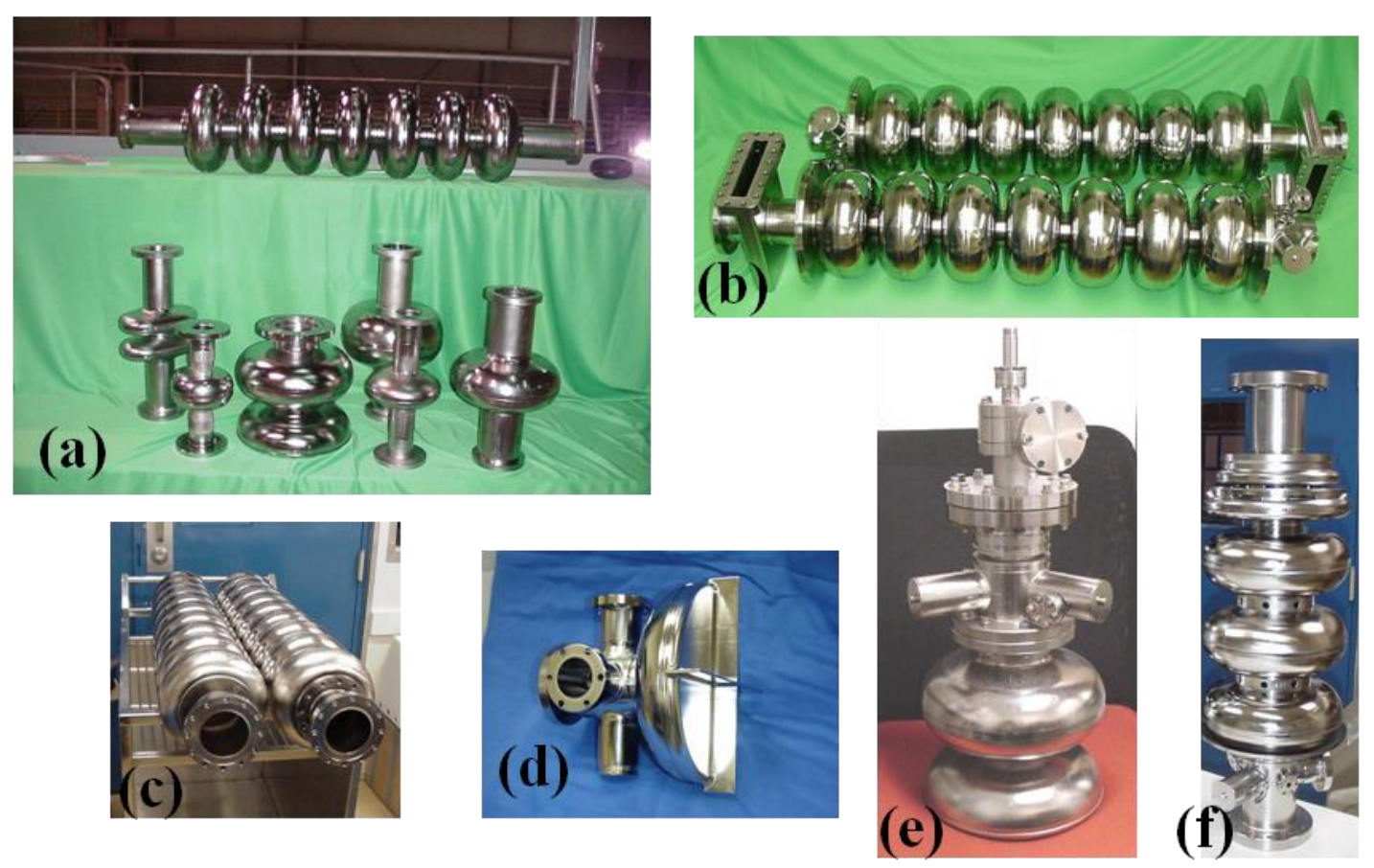

Fig. 10. Collection of cavities (single and multi-cell) fabricated at Jefferson Lab from large-grain/single-crystal material. (a) 7-cell cavity, 2-cell crab, a single-crystal $2.3 \mathrm{GHz}$ LL, a 1.5 cell cavity for joint tests, a $2.3 \mathrm{GHz}$ TESLA-shape single-crystal cavity, (b) two LL and CEBAF Upgrade cavities, (c) two Ichiro-type 9-cell cavities, (d) a half-cell for laser heating experiments, (e) a 1.5 cell photo-injector and (f) a 3.5 cell photo injector cavity of the Rossendorf design.

Experienced cavity manufacturers such as ACCEL Instruments, now Research Instruments, in Germany, built four single-cell and eleven $1.3 \mathrm{GHz}$, nine-cell TESLAtype cavities to date. The half-cells were deep drawn using a stamp and a cushion.

Deviations of the deep-drawn half-cells from the ideal shape were measured with a tactile 3D machine. The distribution of deviations from the ideal shape is slightly broader than that for fine-grain half-cells [71] and grain boundary steps as high as $200 \mu \mathrm{m}$ were measured. However, proper tooling was used to hold the half-cells to the proper shape during the following fabrication step, so that multi-cell cavities with the same tolerances in length, frequencies of the fundamental and higher-order modes as those required for cavities made of fine-grain $\mathrm{Nb}$ were built. No issues with EBW of large-grain material were noticed [80]. To investigate the possible role of grain boundary steps on cavity performance, the half-cells to produce two 9-cell cavities were mechanically polished to remove large steps at grain boundaries. RF test of all 9-cell cavities at $2.0 \mathrm{~K}$ did not show any difference in performance, up to $E_{\text {acc }} \sim 30 \mathrm{MV} / \mathrm{m}$, between cavities with and without polished grain boundaries [71]. Single-crystal cavities were also built. One of the first ingots Jefferson lab received from CBMM had a large central crystal as mentioned earlier. However, this crystal was not large enough for a $1500 \mathrm{MHz}$ CEBAF type cavity; therefore two higher frequency cavities $(2.2 \mathrm{GHz}$ and $2.3 \mathrm{GHz})$ of two different shapes were fabricated without problems. 
The single crystal option was further pursued at DESY and later at Peking university (PKU) based on the DESY proposal described here with the goal to produce cavities of the same frequency as the large-grain cavities (1300 MHz).

The following aspects have been demonstrated at DESY on samples and taken into consideration for the fabrication proposal:

-Definite enlargement of the single crystal disc diameter is possible without destroying the single crystal structure.

-The single crystals keep the crystallographic structure and, after shaping of the cavity half-cell from a disc by deep drawing, the orientation perpendicular to the surface remains.

-Appropriate heat treatment will not destroy the deformed single crystal,

-If the orientation of the crystals is matched, two single crystals will grow together by EB welding.

Especially important for cavity fabrication by EBW are the last three points that allow production of cavities as a complete single crystal. It turned out that two single crystals will grow into one single crystal, if the crystallographic orientations are matched at the EBW seam with an accuracy approaching three degrees.

Some detailed experiments of the single crystal enlargement and subsequent electron beam welding procedures is given in Ref. 16 and confirmed with metallographic and crystallographic investigations on samples by TEM and with SEM

Several single crystal cavities were fabricated in this manner and subsequent performance tests will be described in next section.

\section{Cavity Test Results}

The advent of ingot niobium for SRF cavities had attracted the interest of many institutions and work on fabrication and testing of such cavities was carried out initially at Jefferson Lab, DESY, KEK, Michigan State University (MSU), Cornell University, Peking University (PKU), FermiLab, Institute of High Energy Physics China (IHEP) and Bhabha Atomic Research Centre (BARC), India. In the following the activities in each laboratory will be reviewed.

\subsection{Jefferson Lab, USA}

The evaluation of ingot niobium for SRF cavity application started at Jefferson Lab in 2004 as a collaboration with CBMM. CBMM provided two ingots named " $\mathrm{A}$ " and "B" of approximately $220 \mathrm{lbs}$ each with a diameter of 9.25". Ingot A had a very large single crystal of 7" diameter in its center with a few grains at its periphery, whereas ingot $\mathrm{B}$ had several large grains, but not as large as ingot $\mathrm{A}$. The RRR value of the material was $\sim 280$ with a Ta content of $\sim 800 \mathrm{ppm}$. Post-purification at $1250{ }^{\circ} \mathrm{C}$ in the presence of $\mathrm{Ti}$ did not significantly improve the RRR value $(\sim 10 \%)$. As discussed earlier, the large single crystal of ingot "A" was used to fabricate a $2.2 \mathrm{GHz}$ and a $2.3 \mathrm{GHz}$ single cell cavity. In this phase of exploring the capabilities of large grain ingot material, material from four different manufacturers, as listed in Table 2, was used to fabricate single cell cavities, which subsequently were subjected to the same standard surface treatments such BCP, hydrogen degassing, BCP, high pressure rinsing (HPR) and, "in situ" LTB. Even though the sheets for the half cells were produced in different ways (saw cutting, wire 
EDM) and the material had different grain sizes, RRR values and Ta content, the performances after post-purification were astonishingly very similar as shown in Fig. 11.

Table 2: Properties of ingot niobium supplied by different companies

\begin{tabular}{llll}
\hline Manufacturer & Ta content $(\mathrm{ppm})$ & RRR & Cutting method \\
\hline CBMM & $800-1500$ & 280 & Wire EDM \\
Ningxia & $<150$ & 330 & Saw cutting \\
W. C. Heraeus & $<500$ & 500 & Wire saw \\
Wah Chang & $<500$ & $>300$ & Saw cutting \\
\hline
\end{tabular}

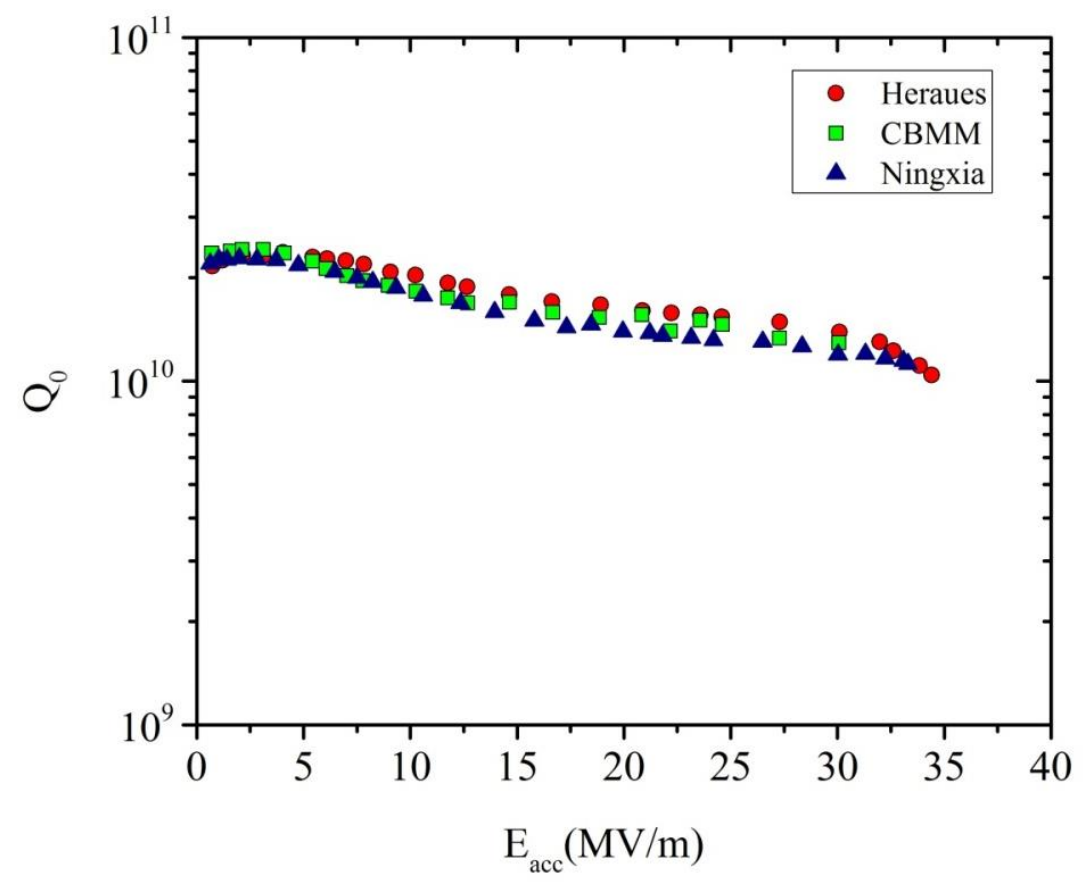

Fig. $11 . Q_{0}$ vs. $E_{a c c}$ at $2.0 \mathrm{~K}$ measured after post-purification at $1250{ }^{\circ} \mathrm{C}$ for $1.3 \mathrm{GHz}$, TESLA-type single-cell cavities made from ingot material from three different vendors after [39]. The ratio $B_{p} / E_{a c c}$ for these cavities is $4.26 \mathrm{mT} /(\mathrm{MV} / \mathrm{m})$.

The comparison of material from different vendors continued thereafter to get better statistics. Five cavities each were fabricated from CBMM, Tokyo Denkai, Ningxia and W.C. Heraeus material and all cavities were subjected to the same surface treatments. This test series started in 2007 with the "standard" surface treatment and initial results have been reported in [81]. Figure 12 shows the best results obtained from W.C. Hereaus, Ningxia and Tokyo Denkai cavities. In some cases the best performance was achieved after the first processing cycle; in other cases an additional high pressure water rinse (HPR) was necessary to eliminate some field emission. Except for TD \#1 all results shown are after LTB, which eliminated the " $Q-$ drop" ( $Q$ degradation at high field). From the set of the CBMM cavities only one cavity was tested with a side-port welded to one half cells at approximately 45 degrees from the equator. This cavity quenched at $E_{a c c} \sim 29$ $\mathrm{MV} / \mathrm{m}$. The remaining CBMM cavities need to go through the testing procedure; also all 20 cavities need to be re-tested after a post-purification heat treatment to complete the 
"statistical" evaluation. This will be done, when the resources are available. For a direct comparison of the material performance one has to look at the peak magnetic surface field at which the quenches in the cavities occurred. Since the cavity from W.C. Heraeus is of the "Low Loss" variety (LL) [73, 82], the highest gradient of $E_{\text {acc }} \sim 45 \mathrm{MV} / \mathrm{m}$ in the LL cavity corresponds to a peak magnetic field of $B_{\text {peak }} \sim 160 \mathrm{mT}$, equivalent to the 35 $\mathrm{MV} / \mathrm{m}$ was reached in the TESLA-type cavities (within 10\%).

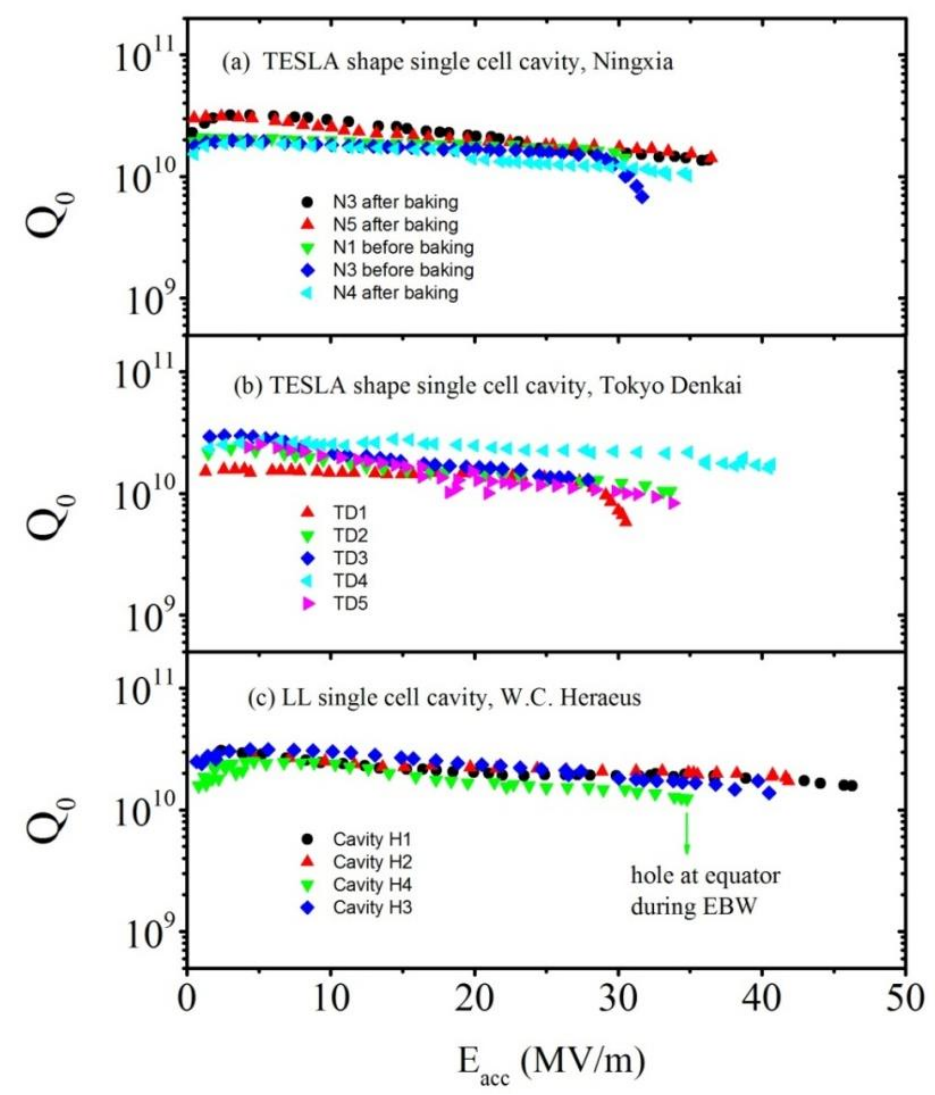

Fig. 12. $Q_{0}\left(E_{a c c}\right)$ measured at $2.0 \mathrm{~K}$ on cavities of different shape made from ingot niobium supplied by three different companies (W.C.Hereaus, Ningxia, Tokyo Denkai) [81]. The ratios of $B_{p} / E_{a c c}$ are $\sim 4.3 \mathrm{mT} /(\mathrm{MV} / \mathrm{m})$ for the TESLA shape and 3.6 $\mathrm{mT} /(\mathrm{MV} / \mathrm{m})$ for the LL shape.

The results on multi-cell cavities are described in Ref. [83] and were limited by repairs in the equator EBW, as mentioned in Sec. 3.3. The highest $B_{\mathrm{p}}$-value of $\sim 130 \mathrm{mT}$, corresponding to $E_{\text {acc }} \sim 31 \mathrm{MV} / \mathrm{m}$, was achieved at $2.0 \mathrm{~K}$ in a $1.3 \mathrm{GHz}$ TESLA-type, 9-cell cavity. The data on multi-cell ingot $\mathrm{Nb}$ cavities showed that the low-field $(\sim 15-20 \mathrm{mT})$ quality factor at $2.0 \mathrm{~K}$ is higher than that of fine-grain ones of the same frequency and with the same treatments, because of a lower residual resistance, as summarized in Fig. 13 , and those higher $Q_{0}$-values can be maintained at higher gradients [84]. 


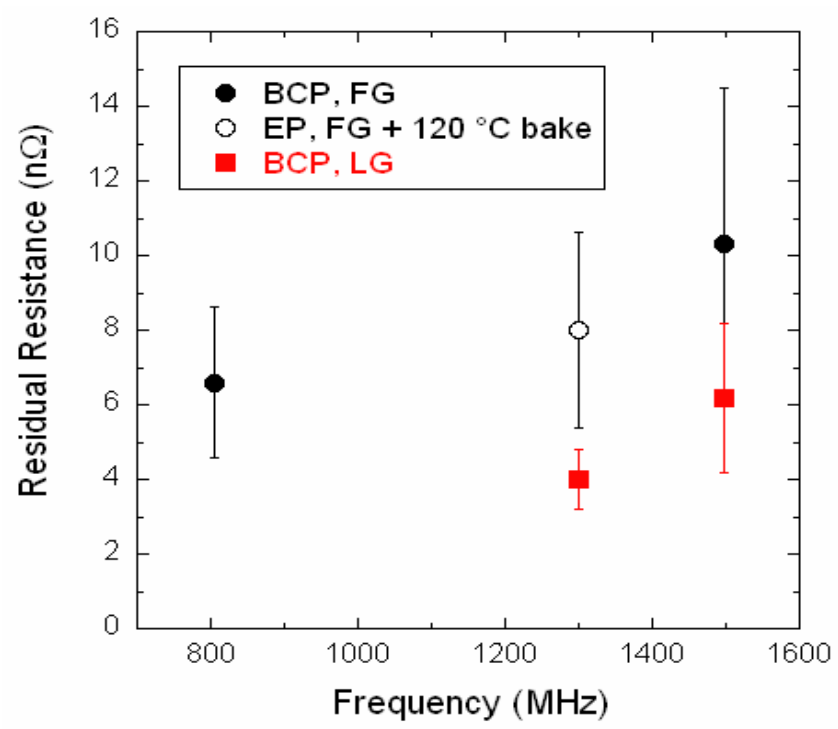

Fig. 13. Residual resistance obtained from low-field $\mathrm{Q}_{0}$-values for different production projects involving many fine-grain cavities and from a more limited number of multi-cell ingot $\mathrm{Nb}$ cavities [83].

A series of tests was carried out with successively removing small amounts of material from the surface and to determine the evolution of the cavity performance as a function of damage layer removal. This series was done with a single crystal cavity of the Tesla shape provided by DESY. A removal of approximately $112 \mu \mathrm{m}$ from the inner surface and only $6 \mathrm{~h}$ of LTB at $120^{\circ} \mathrm{C}$ improved the cavity performance significantly to $E_{a c c}=37.5 \mathrm{MV} / \mathrm{m}$-as shown in Fig. 14 . Further steps of material removal resulted only in a slight improvements of the breakdown field.
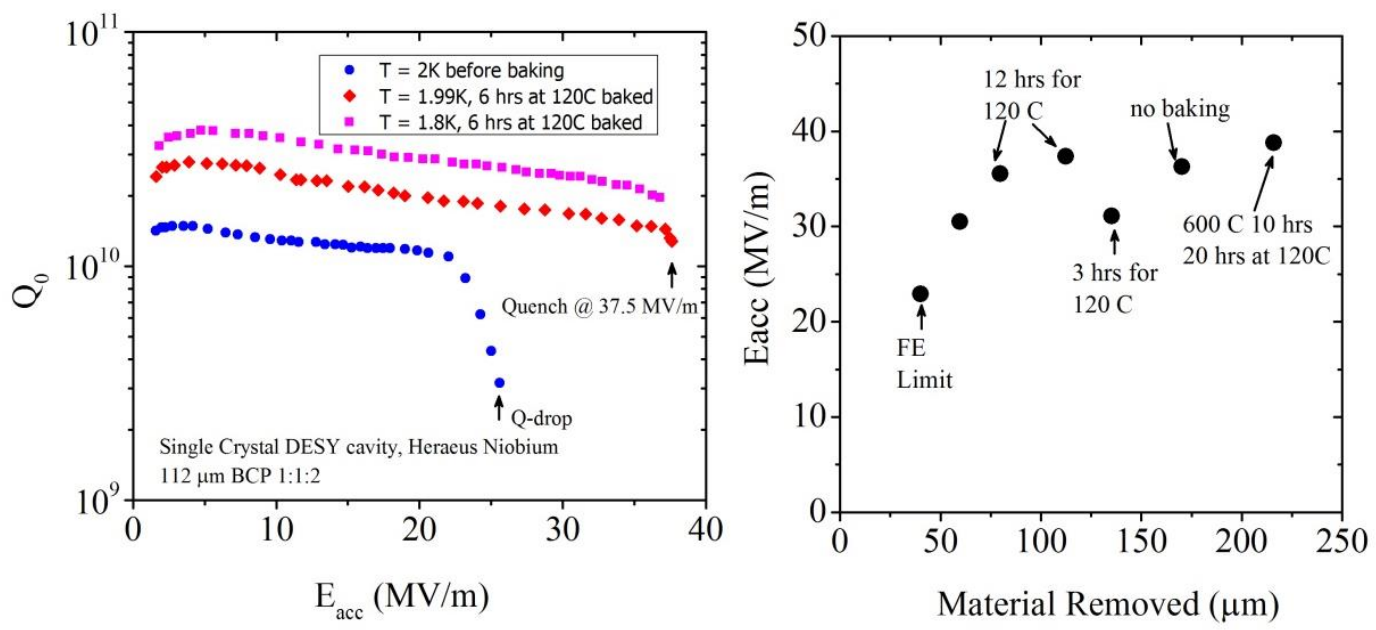

Fig. 14. Performance of a $1.3 \mathrm{GHz}$ a single-crystal single-cell cavity fabricated at DESY as a function of material removal [from ref. 85]. The ratio $B_{p} / E_{a c c}$ for this cavity (TESLA shape) is $4.26 \mathrm{mT} /(\mathrm{MV} / \mathrm{m})$. 


\subsection{DESY, Germany}

The activities and accomplishments at DESY have been summarized in a recent publication [86]. For completion of this review only the highlights of the work at DESY are reported in the following.

Eleven 9-cell 1.3 GHz TESLA-shape cavities for the X-FEL project were built at ACCELL and tested in a vertical cryostat at DESY cavities. The best of these cavities reached a final performance of $E_{a c c}=45.4 \mathrm{MV} / \mathrm{m}$ at a $Q_{0}$-value of $1.3 \times 10^{10}$, outperforming even the highest gradient cavities from fine-grain niobium. This accelerating gradient corresponds to surface magnetic field of $B_{\text {peak }}=192 \mathrm{mT}$. Analysis of mode measurements of the nine pass-band modes showed that in some cells peak surface fields as high as $B_{\text {peak }}=213 \mathrm{mT}$ were reached, which is close to the fundamental limit of bulk niobium. Remarkably, the cavities made from large grain material exhibited higher $Q$-values at the operations gradient of the X-FEL as shown in Figure 15, which also shows the result from the best performing 9-cell cavity.

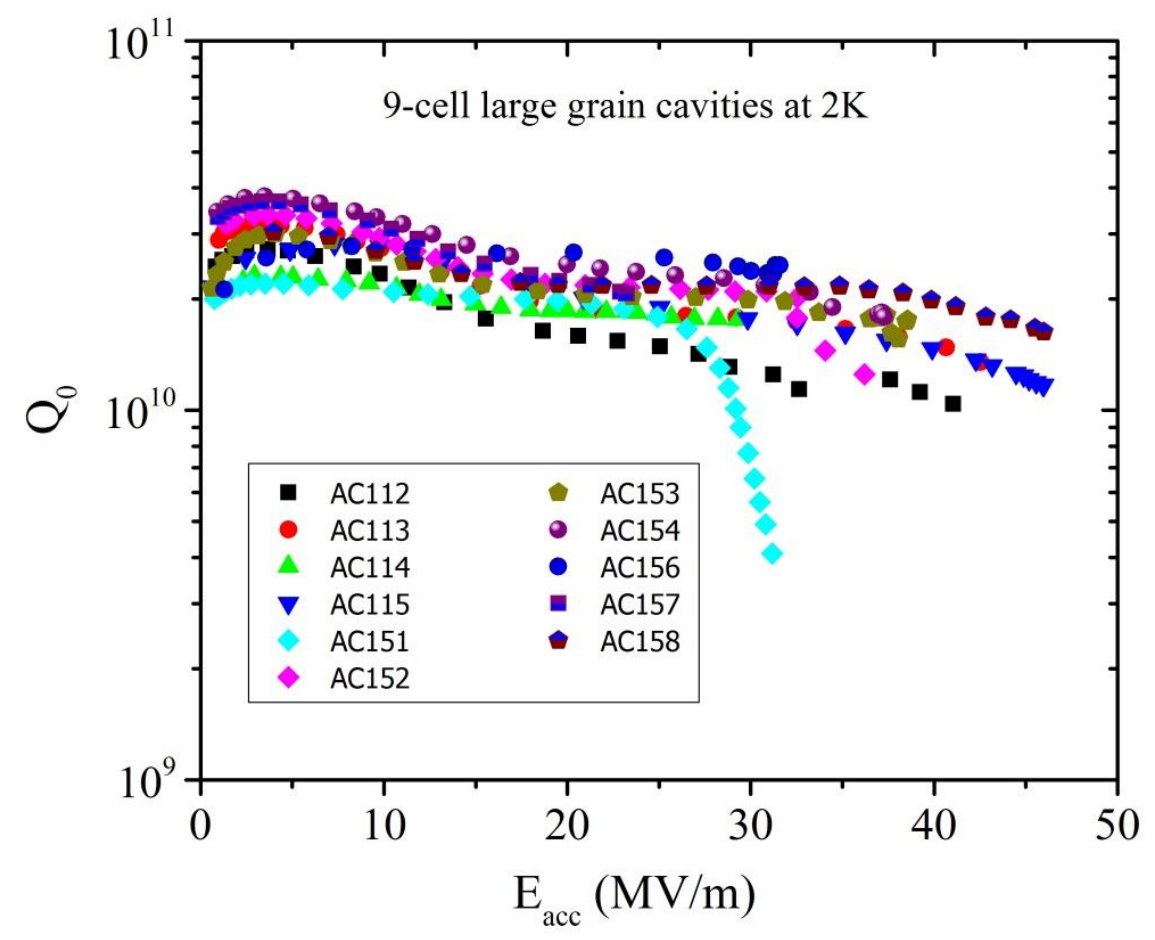

Fig. 15. $Q_{0}\left(E_{a c c}\right)$ for 9-cell cavities $\mathrm{AC} 112-\mathrm{AC} 115, \mathrm{AC} 151-\mathrm{AC} 158$ at $2.0 \mathrm{~K}$, after EP. The ratio $B_{p} / E_{a c c}$ for these cavities is $4.26 \mathrm{mT} /(\mathrm{MV} / \mathrm{m})$.

Higher $Q_{0}$-values by $\sim 25-30 \%$ at $2.0 \mathrm{~K}$ were measured at all gradients, up to $\sim 30$ $\mathrm{MV} / \mathrm{m}$, for 9-cell ingot $\mathrm{Nb}$ cavities compared to fine-grain ones after standard treatments. A significant reduction of the RF heat load for a cryomodule with seven 9-cell ingot $\mathrm{Nb}$ cavities at DESY, compared to that for an identical cryomodule with fine-grain 9-cell cavities, tested under the same conditions, was recently reported, confirming the possibility of obtaining lower values of residual resistance with this material [87]. 


\subsection{High Energy Accelerator Research Organization KEK, Japan}

By 2006 many activities had developed into R\&D programs at KEK with IHEP and Peking University to investigate ingot material from different suppliers. At KEK the initial efforts with ingot material complemented the high-gradient research on fine-grain niobium, which had been pursued for several years in the context of the proposed ILC requirements. This program had focused on developing the Ichiro-type cavity, which differed from the TESLA-type cavity shape by reducing the ratio of the peak magnetic surface field to the accelerating gradient by roughly $15 \%$; this in turn will permit higher accelerating gradients at the same limiting magnetic field as given by the superconducting properties of the material.

The first two single cell cavities were fabricated from CBMM ingot material, followed by three cavities made from Ningxia large grain niobium. Similar fabrication difficulties such as slippage of grain boundaries and non-uniformity in the half cell shapes as in other laboratories were encountered during the fabrication, but did not prevent the completion and testing of the cavities. The same surface preparation steps as developed for fine grain material were applied. The typical process includes the CBP of $\sim 200 \mu \mathrm{m}, 800{ }^{\circ} \mathrm{C}$ for $3 \mathrm{~h}$, light $\mathrm{BCP}$ or $\mathrm{EP}$ removing $\sim 50 \mu \mathrm{m}$ prior to the cryogenic test. The very encouraging result of the first cavity are shown in Fig. 15 together with data from a fine-grain cavity, which had been fabricated and treated in parallel with the cavity from ingot material: the large-grain cavity had a very low residual resistance of $R_{\text {res }}=1.46$ $\mathrm{n} \Omega$; five times lower than measured on the fine-grain cavity. The maximum gradient, however, was limited by quench at a lower field for the ingot $\mathrm{Nb}$ cavity, as shown in Fig. 16 [88].

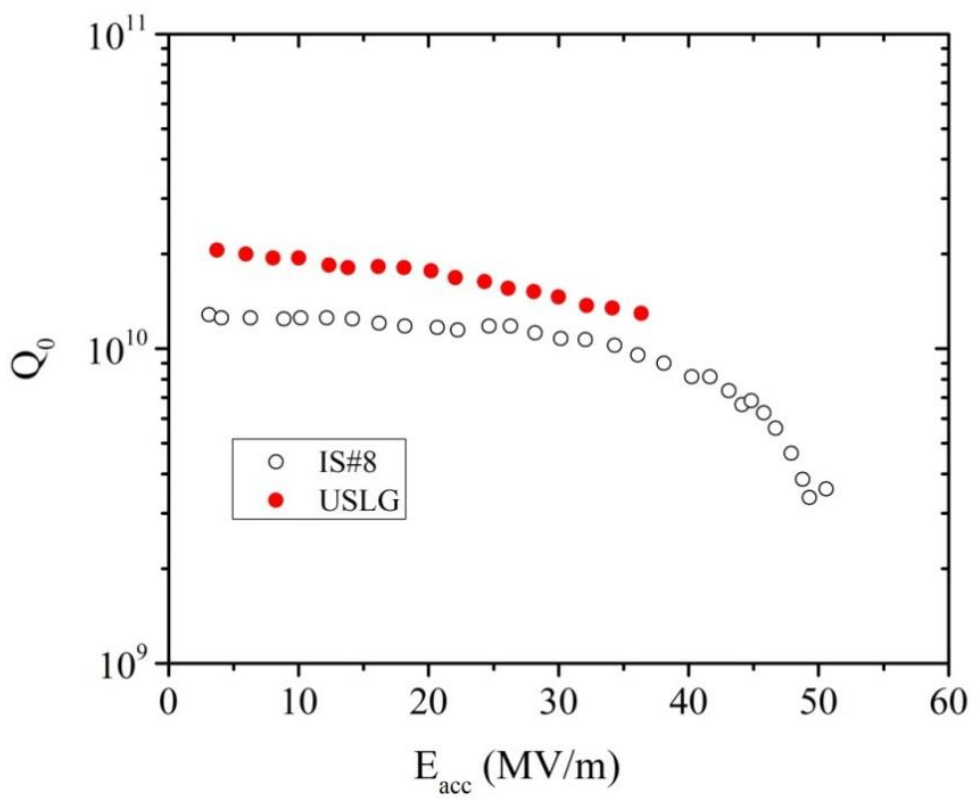

Fig. 16. Performance of the first large grain single cell cavity fabricated at KEK from CBMM ingot niobium [USLG], compared to one of the best fine grain cavities [IS\#8], from ref. 88]. The ratio $B_{p} / E_{a c c}$ for these cavities is $3.56 \mathrm{mT} /(\mathrm{MV} / \mathrm{m})$. 
Three cavities made from Ningxia ingot material were used to investigate several issues:

- How do these cavities perform in comparison to fine grain cavities using KEK standard recipe?

- What is the effect of shorter "in situ" baking times on eliminating Q-drop?

- How does the cavity performance evolve with successive material removal steps by EP after CBP?

- How does the cavity performance evolve with successive EP material removal steps without prior CBP?

This study had the following results:

- The cavities perform very similar to cavities fabricated from fine grain niobium

- The duration of LTB can be reduced from $48 \mathrm{~h}$ for fine-grain cavities to $12 \mathrm{~h}$ to eliminate the high-field $Q$-drop

- A total of $\sim 100 \mu \mathrm{m}$ of EP is necessary for high performance in both $Q$-value and accelerating gradient.

Centrifugal barrel polishing ( $200 \mu \mathrm{m}$ material removal) seems to be essential to achieve high cavity performance; this is mainly due to removing weld irregularities, which have a degrading influence on performance.

A summary of results from the $R \& D$ with the cavities from Ningxia is given in Fig. 17. Both cavities with initial CBP performed very well, reaching gradients of 44 and $48 \mathrm{MV} / \mathrm{m}$, whereas cavity \#3 without CBP showed an early quench limitation.

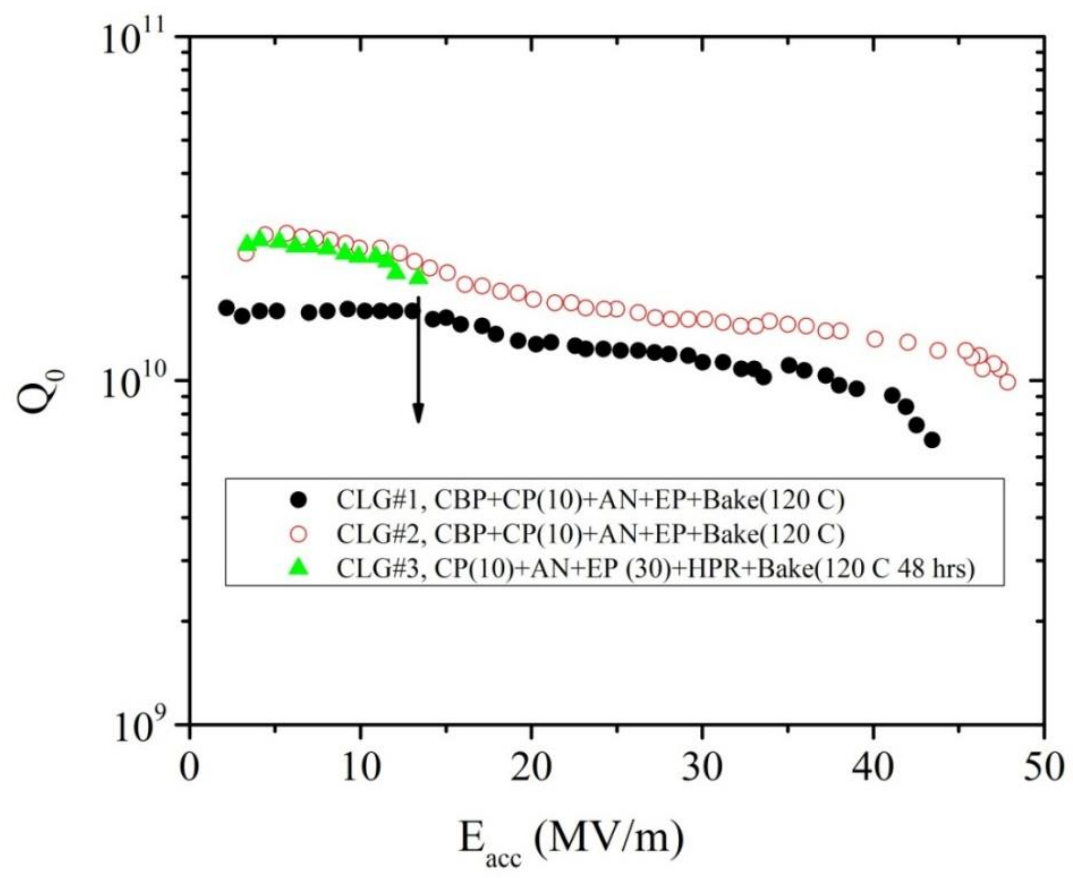

Fig. 17. Summary of the performance of large-grain $1.3 \mathrm{GHz}$ single-cell cavities from Ningxia material. Note: Cavity CLG \#3 did not receive CBP. The nomenclature used in this figure is CBP (centrifugal barrel polishing), $\mathrm{CP}$ (chemical polishing), AN (annealing), EP (electropolishing), HPR (high pressure water rinsing), and baking (120 ${ }^{\circ} \mathrm{C}$ baking) [from ref. 88]. The ratio $B_{p} / E_{\text {acc }}$ for these cavities is $3.56 \mathrm{mT} /(\mathrm{MV} / \mathrm{m})$. 
Subsequent work at KEK concentrated on evaluating ingot material from Tokyo Denkai - the company started production of large grain ingots in 2007 - and on developing in collaboration with industry a multi wire slicing capability based on machines used in the semiconductor industry for wafer slicing. This development has been discussed Sec. 3.1. The single-cell development pursued streamlining of procedures such as the use of BCP instead of EP and investigating the influence of modified procedures such as horizontal BCP. Additionally, reproducibility tests were conducted by repeatedly applying the same surface treatments alternating between horizontal and vertical BCP. In between a test series, the surface was also reset by an additional CBP. These test series indicated that gradients of $E_{a c c} \sim 35 \mathrm{MV} / \mathrm{m}$ can be reliably reached in ICHIRO-type cavities. However gradients of 44-53 MV/m were achieved with fine-grain, electropolished cavities of the same shape [74].

The high gradient program at KEK was designed to demonstrate the capability to achieve ILC gradients of $E_{a c c}=35 \mathrm{MV} / \mathrm{m}$ or higher in 9-cell cavities, initially fabricated from fine grain material. For the ingot niobium program three 9-cell ICHIRO-type cavities were fabricated (I9\#9, I9\#10, and I9 \#11) but only I9\#9 was tested several times. The fabrication process for I9\#10 and I9\#11 incorporated an improved electron beam welding procedure by welding the equators from the inside of the cavity cell. This provides better surface finishes and avoids irregularities in the weld, which often appear if full penetration welds from the outside are used. Presently, only I9\#9 has been tested several times and is limited at $E_{a c c}=27 \mathrm{MV} / \mathrm{m}$ after horizontal BCP [74], this treatment method was developed to achieve better uniformity of material removal and therefore better field flatness in the 9-cell cavity. More tests on the 9-cell cavities are planned for the future.

\subsection{Michigan State University (MSU), USA}

At Michigan State University in collaboration with FermiLab a strong material science program was pursued, focusing on gaining a better understanding of the influence of material properties of niobium and cavity preparation methods on cavity performance. The advent of large grain niobium was welcomed as an additional interesting research topic [27]. The ongoing collaboration between MSU and FNAL also included the design and manufacturing of prototype cavities for the proposed proton driver accelerator, originally planned to be made from fine-grain material. Large-grain niobium opened the possibility of comparing both materials and in collaboration with Jefferson Lab, MSU fabricated two single-cell cavities from CBMM niobium provided by Jefferson Lab. These two cavities were also treated and tested at Jefferson lab, whereas two simultaneously fabricated cavities from fine grain niobium were evaluated at MSU. The results of these efforts are displayed in Fig. 18. Besides the single cell program, two 7cell cavities were fabricated one of each from fine grain and large grain material. The cavities were completed in 2007 but no test results are available yet to the authors' knowledge. 


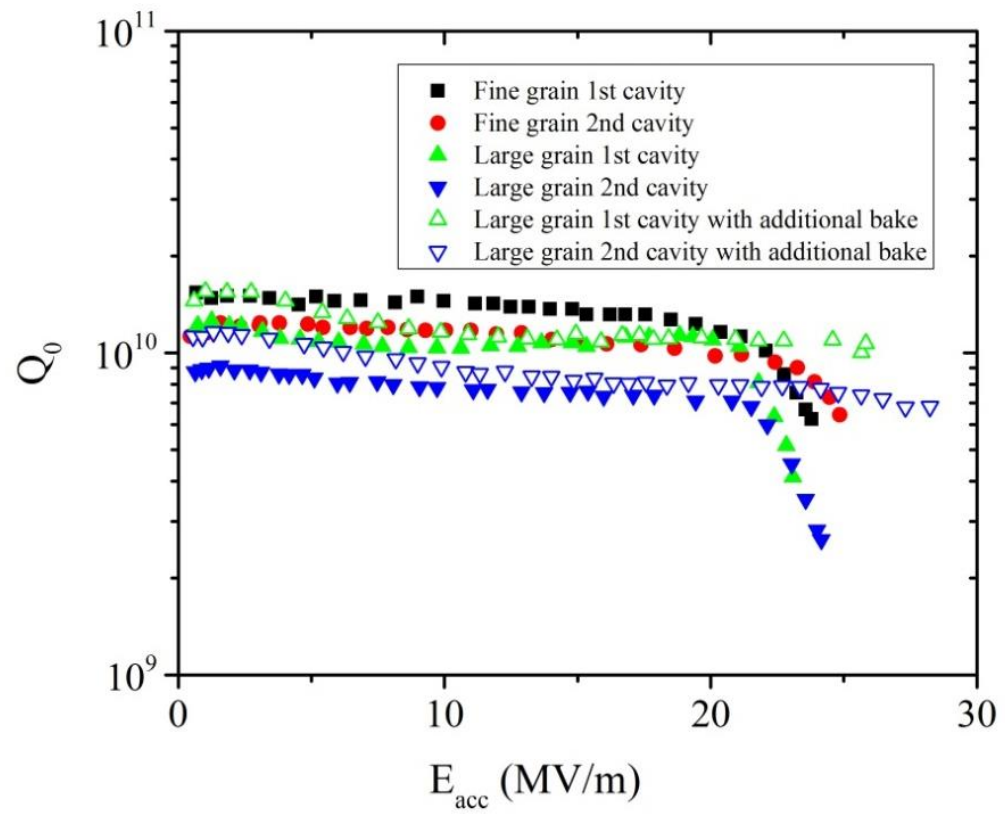

Fig. 18. Test results for single cell cavities: fine grain cavities received BCP and HPR treatment, large grain cavities were post-purified and then BCP'd and high pressure rinsed; both large grain cavities were re-tested after additional bake out [89]. The ratio $B_{p} / E_{a c c}$ for these cavities is $5.27 \mathrm{mT} /(\mathrm{MV} / \mathrm{m})$.

\subsection{Cornell University, USA}

The interest at Cornell University in large-grain niobium centered on the question of the causes for non-linearities in the $Q_{0}$ vs $E_{a c c}$ behavior of a cavity. Therefore, comparative studies were carried out on cavities made from both fine-grain and largegrain niobium using temperature maps. Large-grain material with few, but distinct grain boundaries allows to look at correlations between grain boundary patterns and heating pattern on the outside of the cavity at higher rf fields [47]. The study indicates that there is no correlation between grain boundaries and heated areas: even the breakdown spot is not on a grain boundary, but was identified by optical inspection as a geometrical defect such as a pit. This is an important result reinforcing the notion that grain boundary steps caused by preferential etching are not necessarily causing quench limitations. The temperature maps also showed broader losses in a fine-grain cavity than in a large-grain one [90].

The investigation of the origin of the high-field $Q$-slope was pursued on a finegrain and a large-grain single-cell cavity after BCP [47, 48]. Areas identified as "hotspots" (high RF dissipation) and "cold-spots" in both cavities by temperature mapping were cut and analyzed with electron back-scattered diffraction (EBSD), Auger electron spectroscopy (AES), X-ray photoelectron spectroscopy (XPS) and optical profilometry. The results indicated that no correlation between roughness, oxide structure, grain boundaries and the occurrence of anomalous RF losses was found. However, the distribution of the local mis-orientation angle within grains showed that "cold" samples have lower mis-orientation angle and therefore dislocation density, than "hot" samples as shown in Fig. 19. Hydrogen-dislocation interactions, possibly leading to lossy hydride 
precipitation, could play a role in causing anomalous RF losses as discussed in Refs. [35, $48,57]$.
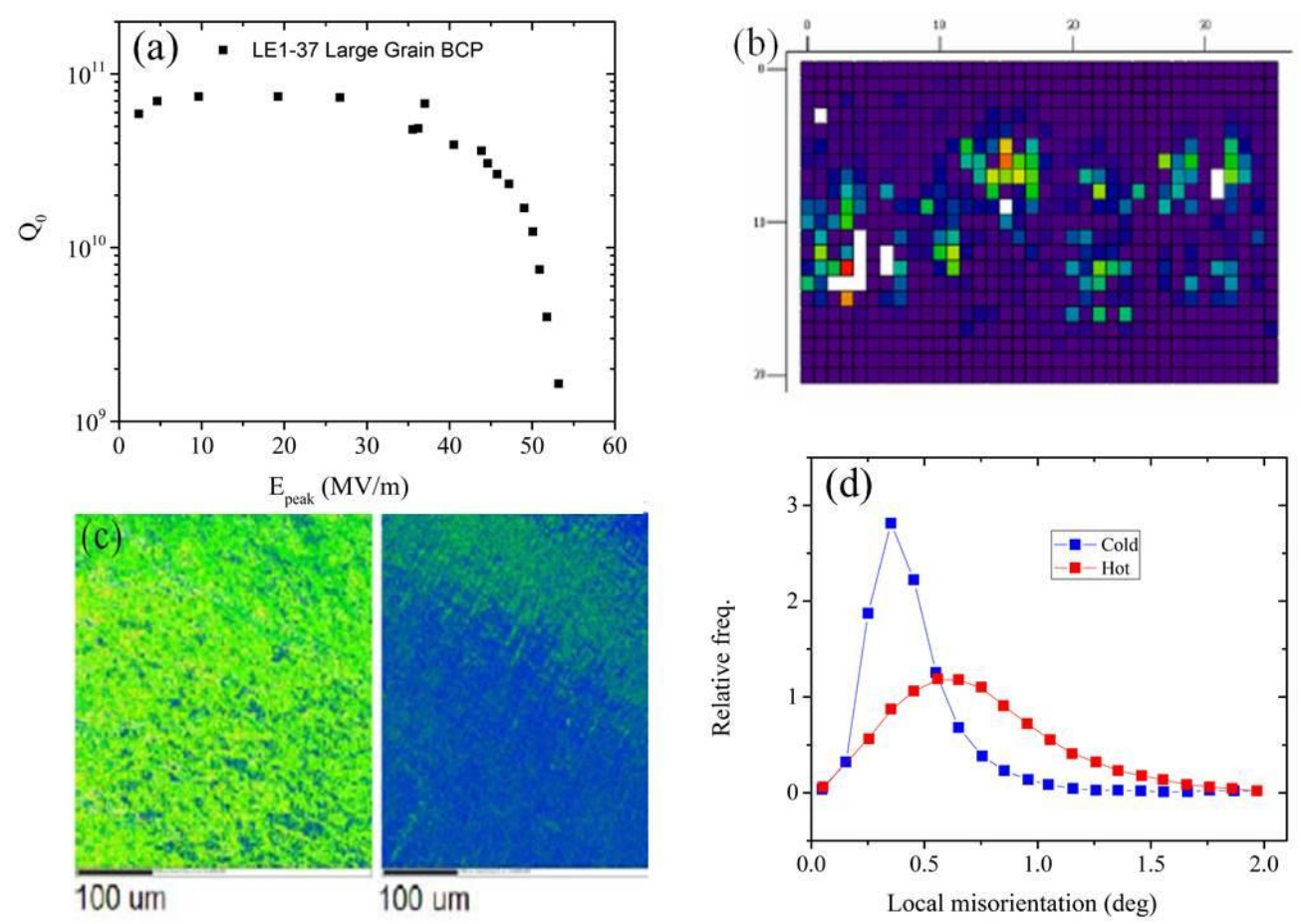

Fig. 19. (a) Performance of $1.5 \mathrm{GHz}$ large-grain cavity, (b) T-map with "hot" and "cold" areas, (c) EBDS map of a hot spot (green) and a cold spot (blue) and (d) distribution of mis-orientation angles in regions of "hot" and "cold" spots [90].

\subsection{Peking University (PKU), China}

Peking University started working on large-grain cavities in 2005 . One $1.3 \mathrm{GHz}$ single-cell and one 2-cell TESLA-shape cavities were fabricated with discs from Ningxia. Both cavities were tested at Jefferson Lab with excellent performances; the single cell cavity reached an accelerating gradient of $E_{a c c}>40 \mathrm{MV} / \mathrm{m}$ with $Q_{0}$ of $\sim 10^{10}$ [91]. Because of this outstanding result some surface inspections were done at FNAL making use of the replica technique developed at this lab [42]. Some of the surface topological features which were seen on the inner cavity surface are shown in Fig. 20 [43]. The presence of a grain boundary step 25-60 $\mu \mathrm{m}$ high at the equator, with an estimated geometric field enhancement factor of 1.6 did not limit the performance of the cavity up to $\sim 185 \mathrm{mT}$.

A 3.5-cell photo-injector cavity was fabricated at PKU from large-grain material and was tested at Jefferson Lab. It reached a gradient of $E_{a c c}=23.5 \mathrm{MV} / \mathrm{m}$, and a $Q_{0}-$ value higher than $1.2 \times 10^{10}$ [92]. All cavities fabricated at PKU achieved respectable results with gradients $E_{a c c}>20 \mathrm{MV} / \mathrm{m}$, however, several equatorial electron beam welds showed defects. It seems likely that with improved electron beam welding the cavities will perform to higher levels. In 2008 PKU produced a single-crystal single-cell cavity using the same enlargement method as developed at DESY as described earlier. The cavity was evaluated at Jefferson Lab. Initially the performance was rather poor due to a sub-standard electron beam weld at the equator. After several iterations of mechanical 
grinding of the weld and subsequent chemical surface treatment by BCP the performance improved significantly and a gradient of $E_{a c c} \sim 29 \mathrm{MV} / \mathrm{m}$ was reached.
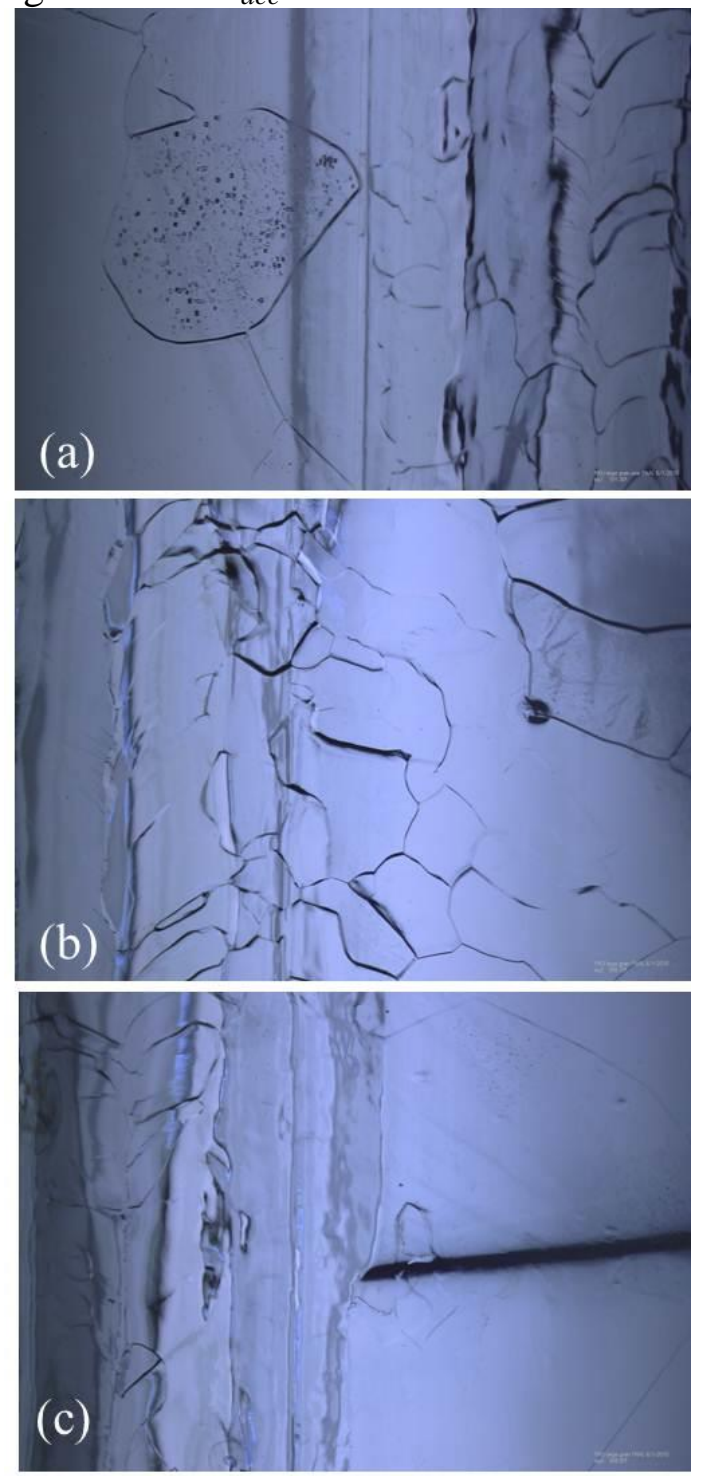

Fig. 20. Optical image of the PKU-LG1 single-cell showing (a) BCP etch pits, (b) a pit in the equatorial weld, and (c) grain boundary step at the equator [43].

\subsection{FermiLab}

At the 2005 Single Crystal/Large Grain International Niobium Workshop a FermiLab program was discussed, which was aiming at two complementary goals: developing local expertise on the fabrication process with ingot $\mathrm{Nb}$ material and to develop an R\&D program to support both large-grain and fine-grain programs to study material properties. Collaboration with MSU was established in 2006/2007 through funding support. Material properties such as mechanical deformations under various external forces and of different crystal orientations as well as thermal properties of different material conditions were studied (see above). Also, as part of this collaboration several cavities were fabricated at MSU, but not all of them could be tested. 


\section{8 Institute of High Energy Physics (IHEP), China}

IHEP started R\&D on $1.3 \mathrm{GHz}$ large-grain cavities in 2006. Three electropolished ICHIRO single-cell cavities were fabricated and processed in KEK with Ningxia large-grain niobium material provided by IHEP. The maximum gradient achieved was 47.9 MV/m [93]. Later two LL-type single-cell cavities were fabricated at IHEP and tested at KEK which reached the accelerating gradient of $\sim 40 \mathrm{MV} / \mathrm{m}$ [94]. The first 9-cell cavity IHEP-01 was fabricated and processed at IHEP and tested at the KEK. The maximum gradient reached $\sim 20 \mathrm{MV} / \mathrm{m}$ [95].

\subsection{Bhabha Atomic Research Centre (BARC), India}

Bhabha Atomic Research Centre (BARC) recently started the activities on cavity fabrications targeting the development of superconducting accelerator technology for the accelerator driven subcritical system. A single cell cavity with the resonance frequency of $1050 \mathrm{MHz}, \beta=0.49$ was fabricated from the ingot provided by CBMM with RRR $\sim 96$. The cavity was tested at Jefferson Lab and quenched at an accelerating gradient of $\sim 6$ $\mathrm{MV} / \mathrm{m}\left(B_{p} \sim 48 \mathrm{mT}\right)$ with low-field $Q_{0}$ of $\sim 10^{10}$ [96]. This "sub-standard" performance is suspected to be caused by a large number of etch pits observed after the chemical etching.

\section{DISCUSSION}

The development of ingot $\mathrm{Nb}$ technology over the past decade provided an opportunity to further study the scientific and technical aspects related to SRF cavity made of bulk $\mathrm{Nb}$. There are at the present two applications for superconducting cavities in accelerators:

- Continuous-wave $(\mathrm{CW})$ accelerators, such as energy recovery linacs or proton linacs where only moderate gradients $\left(E_{\text {acc }} \leq 20 \mathrm{MV} / \mathrm{m}\right)$ are required but high $Q_{0^{-}}$ values are needed to reduce the cryogenic losses.

- Pulsed, high-energy accelerators such as ILC which requires very high $\left(E_{a c c}>30\right.$ $\mathrm{MV} / \mathrm{m}$ ) accelerating gradients.

For SRF technology these applications present several challenges such as:

- Understand the causes of RF losses and in particular understand and eliminate "residual losses".

- Understand the causes for "premature" quenches in a cavity and eliminate the causes.

- Understand the non-linear behavior of RF to maintain high efficiency up to the highest gradients.

The main feature of ingot $\mathrm{Nb}$ material is that the total length of grain boundaries is reduced by orders of magnitude compared to standard fine-grain material. Grain boundaries represent areas of reduced superconductivity due to oxidation and segregation of impurities, causing them to behave as "weak links". They are a well-known limitation of thin-film high-temperature superconductors, causing high residual losses and nonlinearities in the field dependence of the surface resistance [97]. Grain boundaries present a limitation for $\mathrm{Nb}$ thin-films as well [98]. However, sample measurements indicate that grain boundaries of bulk $\mathrm{Nb}$ after standard treatments are relatively "clean" and SRF cavity measurements did not reveal any significant limitation posed by grain boundaries to the RF performance. 
Samples measurements also indicated that ingot $\mathrm{Nb}$ has weaker pinning, causing a lower fraction of the residual DC ambient field to be trapped in the material when crossing the transition temperature, compared to fine-grain material. Lower density of pinned fluxoids would result in reduced residual RF losses, which have been observed in many large-grain cavities compared to fine-grain cavities after similar standard treatments. This would be an advantage of ingot $\mathrm{Nb}$ compared to fine-grain $\mathrm{Nb}$ for $\mathrm{CW}$ accelerator applications. Another possible mechanism for RF dissipation at grain boundaries is the penetration of mixed Abrikosov-Josephson fluxoids at relatively high fields $[99,100]$. It should also be mentioned that recent advances in R\&D of treatment of bulk Nb SRF cavities at Jefferson Lab [101] and Fermi Lab [102] lead to the achievement of significantly reduced BCS losses by impurity doping, resulting in very high $Q_{0^{-}}$-values for both fine-grain and large-grain cavities.

Surface studies to characterize oxides, impurities and electronic density of states did not show any significant difference between large-grain and fine-grain $\mathrm{Nb}$ and understanding the impact of such surface properties to the RF performance of cavities is a common challenge for both types of material [103]. Sample measurements showed that very smooth surfaces (less than $100 \mathrm{~nm}$ RMS roughness) within grains of ingot $\mathrm{Nb}$ material can be achieved by simple BCP, compared to EP-treated fine-grain. This was shown to be beneficial for reducing particulate contamination and increase the onset of field emission. The occurrence of steps at grain boundaries after BCP does not seem to limit the cavity performance.

RF dissipation causes the temperature of the inner cavity surface to increase with increasing RF, which cause dissipation to increase even further due to a positive thermal feedback. This phenomenon contributes to the reduction of $Q_{0}$ with increasing field typically observed in cavities after standard treatments. The presence of a phonon peak in the thermal conductivity at $\sim 2 \mathrm{~K}$ measured on large-grain samples, compared to finegrain ones, can reduce the effect of thermal feedback by increased thermal conduction. Some large-grain cavities show a remarkably "flat" $Q_{0}\left(E_{\text {acc }}\right)$ curve but the relation between this behavior and increased thermal conductivity, compared to fine-grain cavities, requires further systematic studies.

Slicing techniques have been developed to cut slices from $\mathrm{Nb}$ ingots with the required tolerance and surface finish, in a cost-effective way. The fabrication of cavities from large-grain $\mathrm{Nb}$ posed some challenges due to the anisotropic deformation of largecrystals of different orientation, as discussed in Sec. 3.3. However, at least one industrial manufacturer has demonstrated that multi-cell cavities can be built with the same stringent tolerances of fine-grain cavities and without any cost increase. Cryogenic tests of cavities proved that grain boundaries of ingot $\mathrm{Nb}$ cavities are leak tight. Another potential issue of ingot $\mathrm{Nb}$ cavities is related to their mechanical stability at room temperature and cryogenic temperatures. The operation of two large-grain cavities inside cryomodule operating in the FLASH accelerator at DESY presented no issues. 9-cell cavities made of ingot $\mathrm{Nb}$ had also been qualified to meet the "pressure vessel" code and seven of them have been installed in a cryomodule for the X-FEL project at DESY [104].

Finally, regarding the cost of the material, there is a reasonable expectation that the reduction of waste and processing steps required to fabricate discs from ingot $\mathrm{Nb}$ compared to the fabrication of fine-grain sheets might lower cost for ingot $\mathrm{Nb}$ material. 


\section{CONCLUSION}

Ingot niobium was re-introduced in 2004 as the result of collaboration between Jefferson Lab and CBMM, the largest niobium producing companies in the world. The "excitement" for this material was generated by the potential of producing similar, if not better, performance levels in SRF cavities as with fine-grain niobium at a lower price. The introduction of ingot niobium into the SRF community has generated an enormous amount of interest, especially in the material and surface science communities, to explore its potential benefit as an alternate material to fabricate bulk Nb SRF cavities. The results of such efforts, which we have attempted to summarize in this review, have shown that ingot $\mathrm{Nb}$ technology is ready to be adopted for an accelerator project. By applying standard cavity processing steps, the highest accelerating gradient in a multi-cell cavity has been achieved with ingot niobium, corresponding to a surface magnetic field of $B_{\text {peak }} \sim 192 \mathrm{mT}$, close to the theoretical critical field of niobium, and statistically higher $Q_{0}$-values compared to fine-grain cavities have been measured.

CBMM has expanded their electron beam melting furnace capability and is now able to produce ingots of $520 \mathrm{~mm}$ diameter, sufficient to produce discs which can be used to fabricate cavities with frequency as low as $650 \mathrm{MHz}$. Qualification of industrial companies, besides Research Instruments, to manufacture ingot $\mathrm{Nb}$ cavities is necessary to make ingot $\mathrm{Nb}$ technology ready for large-scale accelerator projects, such as the proposed Linear Collider. 


\section{ACKNOWLEDGEMENTS}

The authors from Jefferson Lab would like to thank T. Carneiro of CBMM for his enthusiastic support of the development of this technology in the initial stages and also Dr. Swapan Chattopadhyay, the former director of the accelerator division at Jefferson lab for his far sighted view of these activities and his important support. 
*kneisel@jlab.org

\# rao@jlab.org

\section{REFERENCES}

[1] G. M. Mellors, S. Sanderoff, J. Electrochem. Soc. 112 (1965) 2669.

[2] B. Hillenbrand, H. Diepers, Technical Report NT124, Siemens AG (1970).

[3] J. P. Turneaure, I. Weissman, J. Appl. Phys. 39 (1968) 4417.

[4] I. Weissman, J. P. Turneaure, Appl. Phys. Lett 13 (1968) 390.

[5] J. P. Turneaure, Adv. Cryogenic Eng. 13 (1967) 333.

[6] J. P. Turneaure, N. T. Viet, Appl. Phys. Lett. 16 (1970) 333.

[7] P. Kneisel, Joint CERN-Karlsruhe Symposium on SC RF Seperators, Kernforschungszentrum Karlsruhe, Germany, KfK Reports 3/69-19 (1969).

[8] H. Pfister, Cryogenics, 16 (1976) 17.

[9] J. P. Turneaure in Proceedings of the 1971 Particle Accelerator Conference, Chicago, IL, 1971, edited by F. T. Howard (IEEE, 1971), pp. 166-167.

[10] www.jacow.org.

[11] Proceedings of the international Niobium Workshop on Single Crystal-Large Grain niobium technology, Araxá, Brazil, edited by G. R. Myneni, T. Carneiro and A. Hutton, AIP Conf. Proc. 927 (2006).

[12] Proceeding of Symposium on the Superconducting Science and Technology of Ingot Niobium, Newport News VA, edited by G. R. Myneni, G. Ciovati and M. Stuart, AIP Conf. Proc. 1352 (2010).

[13] G. R. Myneni, in: Proc. of the International Niobium Workshop on Single CrystalLarge Grain niobium technology, Araxá, Brazil, AIP Conference Proc. 927 (2006) 41.

[14] R. E. Ricker, D. J. Pitchure, G. R. Myneni, in: Proc. of the International Niobium Workshop on Single Crystal-Large Grain niobium technology, Araxá, Brazil, AIP Conference Proc. 927 (2006) 60.

[15] T. Gnaeupel-Herold et al., in: Proc. of the International Niobium Workshop on Single Crystal-Large Grain niobium technology, Araxá, Brazil, AIP Conference Proc. 927 (2006) 48.

[16] A. Ermakov et al., J. of Physics: Conference Series 97 (2008) 012014.

[17] T. R. Bieler et al., Phys. Rev. ST Accel. Beams 13 (2010) 031002.

[18] T. S. Byun, S. H. Kim and J. Mammosser, J. Nucl. Mat. 392 (2009) 420.

[19] S. K. Chandrasekaran, Role of metallurgy in the thermal conductivity of superconducting niobium, $\mathrm{PhD}$ Dissertation, MSU, 2013.

[20] P. Dhakal, G. Ciovati, G. R. Myneni, P. Kneisel, in: Proc. of the 15th International Conference on RF Superconductivity, Chicago, IL, July 2011, p. 856.

[21] S. K. Chandrasekaran et al., Recovery of Phonon Peak in Annealed Niobium as a Function of Initial Strain and Hydrogen Concentration, $7^{\text {th }}$ SRF Materials Workshop, Newport News, VA, July 16-17, 2012. https://www.jlab.org/indico/conferenceDisplay.py?confId=20.

[22] S. Casalbuoni et al., in: Proc. of the 11th International Conference on RF Superconductivity, Hamburg, Germany, September 2003, p. 555. 
[23] Z. H. Sung, The influence of grain boundaries on the properties of superconducting radio frequency cavity niobium, PhD Thesis, University of Wisconsin, Madison, 2010.

[24] A. Dhavale, P. Dhakal, A. A. Polyanski and G. Ciovati, Supercond. Sci. and Technol. 25 (2012) 065014.

[25] S. B. Roy, G. R. Myneni, and V. C. Sahni, Supercond. Sci. Technol. 22 (2009) 105014.

[26] D. Kang, D. C. Baars, T. R. Bieler and C. C. Compton, in: Proc. of Symposium on the Superconducting Science and Technology of Ingot Niobium, Newport News VA, AIP Conf. Proc. 1352 (2010) 90.

[27] C. Compton, et al., in: Proc. of the international Niobium Workshop on Single Crystal-Large Grain niobium technology, Araxá, Brazil, AIP Conf. Proc. 927 (2006) 98.

[28] P. J. Lee et al., in: Proc. of the Workshop on Pushing the Limits of RF Superconductivity, Argonne, IL, March 2005, ANL Report 05/10, p. 281.

[29] A. A. Polyanskii et al., in: Proc. of Symposium on the Superconducting Science and Technology of Ingot Niobium, Newport News VA, AIP Conf. Proc. 1352 (2010) 186.

[30] P. J. Lee et al., Physica 441 (2005) 126.

[31] A. Grassellino et al., Phys. Rev. ST Accel. Beams 16 (2013) 062002.

[32] S. Aull et al., Phys. Rev. ST Accel. Beams 15 (2012) 062001.

[33] J.-M. Vogt, O. Kugeler, and J. Knobloch, Phys. Rev. ST Accel. Beams 16 (2013) 102002.

[34] A. D. Batchelor et al., in: Proc. of the International Niobium Workshop on Single Crystal-Large Grain niobium technology, Araxá, Brazil, AIP Conference Proc. 927 (2006) 72.

[35] F. Barkov, A. Romanenko, Y. Trenikhina, A. Grassellino, J. Appl. Phys. 114 (2013) 164904.

[36] A. Dangwal, Enhanced field emission from metallic surfaces and nanowires, Ph.D. Dissertation, University of Wuppertal, Germany, 2007.

[37] A. D. Pandey, G. Müller, D. Reschke and X. Singer, Phys. Rev. ST Accel. Beams 12 (2009) 023501.

[38] A. Navitzky, et al., Phys. Rev. ST Accel. Beams 16 (2013) 12001.

[39] P. Kneisel et al., in: Proc. of the International Niobium Workshop on Single CrystalLarge Grain niobium technology, Araxá, Brazil, AIP Conference Proc. 927 (2006) 84.

[40] C. Xu, H. Tian, C. E. Reece and M. J. Kelley, Phys. Rev. ST Accel. Beams 14 (2011) 123501.

[41] C. Xu, H. Tian, C. E. Reece and M. J. Kelley, Phys. Rev. ST Accel. Beams 15 (2012) 043502.

[42] M. Ge et al., Supercond. Sci. and Techn. 24 (2011) 035002.

[43] M. Ge, G. Wu, P. Kneisel, K. Zhao, Investigation of large grain cavity surfaces, presented at the Symposium on Superconducting Science and technology of Ingot Niobium, Newport News, VA, September 2010.

http://www.jlab.org/conferences/sstin/program.html. 
[44] X. Zhao, G. Ciovati and T. R. Bieler, Phys. Rev. ST Accel. Beams 13 (2010) 124701.

[45] T. Proslier et al., Appl. Phys. Lett. 92, (2008) 212505.

[46] T. Proslier et al., in: Proc. of the 14th International Conference on RF Superconductivity, Berlin, Germany, September 2009, p. 137.

[47] A. Romanenko, H. Padamsee, Supercond. Sci. Technol. 23 (2010) 045008.

[48] G. Eremeev, H. Padamsee, in: Proc. of the $10^{\text {th }}$ European Particle Accelerator Conference, Edinburgh, UK, June 2006, p. 475.

[49] G. Ciovati, G. Myneni, F. Stevie, P. Maheshwari, D. Griffis, Phys. Rev. ST Accel. Beams 13 (2010) 022002.

[50] P. Maheshwari, Surface Characterization of impurities in superconducting niobium for radio frequency (RF) cavities used in particle accelerators, $\mathrm{PhD}$ Dissertation, North Carolina State University, NC, 2012.

[51] A. Romanenko, L. V. Goncharova, Supercond. Sci. and Technol. 24 (2011) 105017.

[52] J. F. Zasadzinski et al., in: Proc. of the 15th International Conference on RF Superconductivity, Chicago, IL, July 2011, p. 912.

[53] L. de Moura, C. A. de Faria Sousa, E. B. Cruz, in: Proc. of Symposium on the Superconducting Science and Technology of Ingot Niobium, Newport News VA, AIP Conf. Proc. 1352 (2010) 69.

[54] H. Tian, Surface oxide studies on solid Niobium for Superconducting RF Accelerators using variable photon energy XPS, SRF Materials Workshop, FermiLab, May 23-24, 2007, http://tdserver1.fnal.gov/project/workshops/RF_Materials/agenda.htm

[55] R. L. Paul et al., Materiaux \& Techniques 7-8-9 (2003) 23.

[56] G. Ciovati, J. Appl. Phys. 96 (2004) 1591.

[57] A. Romanenko, F. Barkov, L. D. Cooley, A. Grassellino, Supercond. Sci. and Technol. 26 (2013) 035003.

[58] E. Mahner et al., in: Proc. of the 6th International Conference on RF Superconductivity, Newport News, VA, October 1993, p. 1085.

[59] G. Ciovati, P. Kneisel, in Proc. of the 2006 Linear Accelerator Conference, Knoxville, TN p. 324.

[60] G. Ciovati, P. Kneisel, G. R. Myneni, S. Chattopadhyay, in Proceedings of the 2006 Linear Accelerator Accelerator Conference, Knoxville, TN, p.318.

[61] P. Kneisel, in: Proc. of the 11th International Conference on RF Superconductivity, Hamburg, Germany, September 2003 p. 206.

[62] G. Ciovati et al., Proc. of the 15th International Conference on RF Superconductivity, Chicago, IL, July 2011 p. 319.

[63] S. B. Roy, G. R. Myneni, and V. C. Sahni, Supercond. Sci. Technol. 21 (2008) 065002.

[64] J. Halbritter, in Proc. of the 11th International Conference on RF Superconductivity, Hamburg, Germany, September 2003 p. 156.

[65] T. Proslier, et al., IEEE Trans. Appl. Supercond. 19 (2009) 1404.

[66] Ph. Niedermann, N. Sankarraman, O. Fischer, Proc. of the $2^{\text {nd }}$ Workshop on RF Superconductivity, Geneva, Switzerland, July 1984 p. 583. 
[67] W. Singer et al., in: Proc. of the 14th International Conference on RF Superconductivity, Berlin, Germany, September 2009 p. 411.

[68] W. Singer, X. Singer, Technical Specifications for Large Crystal Niobium Discs Applied for the Fabrication of 1.3 GHz Superconducting Cavities, version B, DESY April 2006.

[69] R. A. Graham, in: Proc. of the International Niobium Workshop on Single CrystalLarge Grain niobium technology, Araxá, Brazil, AIP Conference Proc. 927 (2006) 191.

[70] H.Umezawa et al., in: Proceedings of the 1st International Particle Accelerator Conference, Kyoto, Japan, May 2010, p. 438.

[71] W. Singer et al., in: Proc. of the International Niobium Workshop on Single CrystalLarge Grain niobium technology, Araxá, Brazil, AIP Conference Proc. 927 (2006) 123.

[72] K. Saito et al., in: Proc. of the 14th International Conference on RF Superconductivity, Berlin, Germany, September 2009, p. 467.

[73] J. Sekutowicz et al., in: Proc. of the 2003 Particle Accelerator Conference, Portland, OR, May 2003 p.1395.

[74] F. Furuta, K. Saito, T. Konomi, : Proc. of the International Niobium Workshop on Single Crystal-Large Grain niobium technology, Araxá, Brazil, AIP Conference Proc. 927 (2006) 169.

[75] T. Kubo, et al., in: Proc. of the 2014 International Particle Accelerator Conference, Dresden, Germany, p. 2519.

[76] A. Brinkmann, et al. in: Proc. of the $8^{\text {th }}$ Workshop on RF Superconductivity, Abano Terme, Italy, October 1997, p. 452.

[77] R. Geng, et al., in: Proc. of the $13^{\text {th }}$ Workshop on RF Superconductivity, Beijing, China, October 2007, p. 525.

[78] B. Aune et al., Phys. Rev. ST Accel. Beams 3092001 (2000).

[79] H. Wang, R. A. Rimmer, G. Wu, in: Proc. of the 2005 Particle Accelerator Conference, Knoxville, TN p. 4191.

[80] M. Pekeler et al., in: Proc. of the International Niobium Workshop on Single Crystal-Large Grain niobium technology, Araxá, Brazil, AIP Conference Proc. 927 (2006) 141.

[81] P. Kneisel, in: Proc. of the $13^{\text {th }}$ Workshop on RF Superconductivity, Beijing, China, October 2007 p. 728.

[82] J. Sekutowicz et al., in: Proc. of the 2005 Particle Accelerator Conference, Knoxville, TN, p. 3342.

[83] G. Ciovati, P. Kneisel, G. R. Myneni, in: Proc. of Symposium on the Superconducting Science and Technology of Ingot Niobium, Newport News VA, AIP Conf. Proc. 1352 (2010) 25.

[84] R. Geng et al., in: Proc. of the 15th International Conference on RF Superconductivity, Chicago, IL, July 2011 p. 501.

[85] W. Singer, X. Singer, P. Kneisel, in: Proc. of the International Niobium Workshop on Single Crystal-Large Grain niobium technology, Araxá, Brazil, AIP Conference Proc. 927 (2006) 133.

[86] W. Singer et al., Phys. Rev. ST Accel. Beams 14 (2013) 123501. 
[87] J. Sekutowicz, Material activities towards high $\mathrm{Q}_{0}$ at DESY, TESLA Technology Collaboration Meeting, Hamburg, Germany, March 24-27, 2014.

[88] K. Saito et al., in: Proc. of the International Niobium Workshop on Single CrystalLarge Grain niobium technology, Araxá, Brazil, AIP Conference Proc. 927 (2006)151.

[89] W. Hartung et al., in: Proc. of the $13^{\text {th }}$ Workshop on RF Superconductivity, Beijing, China, October 2007, p. 255.

[90] A. Romanenko et al., in: Proc. of the $13^{\text {th }}$ Workshop on RF Superconductivity, Beijing, China, October 2007, p. 173.

[91] J. Chen, K. Zhao, in: Proc. of the $13^{\text {th }}$ Workshop on RF Superconductivity, Beijing, China, October 2007, p. 1.

[92] S. Quan, et al., Phys. Rev. ST Accel. Beams 13 (2010) 042001.

[93] Z. G. Zong et al., in: Proc. of the 2007 Particle Accelerator Conference, Albuquerque, New Mexico, p. 2426.

[94] Z. G. Zong et al., in: Proceedings of the 2008 European Particle Accelerator Conference, Genoa, Italy, p. 943.

[95] J. Gao et al., in: Proceedings of the 2008 Linear Accelerator Conference, Tsukuba, Japan, p. 1022.

[96] J. Mondal et al., J. Instrumentation 6 (2011) T11003.

[97] A. M. Portis, Electrodynamics of high-temperature superconductors, Lecture notes in Physics 48, World Scientific, Singapore, 1993.

[98] J. Halbritter, J. Appl. Phys. 97 (2005) 083904.

[99] A. Gurevich and L. D. Cooley, Phys. Rev. B 50 (1994) 13563.

[100] A. Gurevich, Phys. Rev. B 65 (2002) 214531.

[101] P. Dhakal et al., Phys. Rev. ST Accel. Beams 16 (2013) 042001.

[102] A. Grassellino et al., Supercond. Sci. Technol. 26 (2013) 102001.

[103] A. Gurevich, Rev. Accel. Sci. Technol. 5 (2012) 119.

[104] W. Singer, Cavity Material for the European XFEL, TESLA Technology Collaboration Meeting, Hamburg, Germany, March 24-27, 2014. 